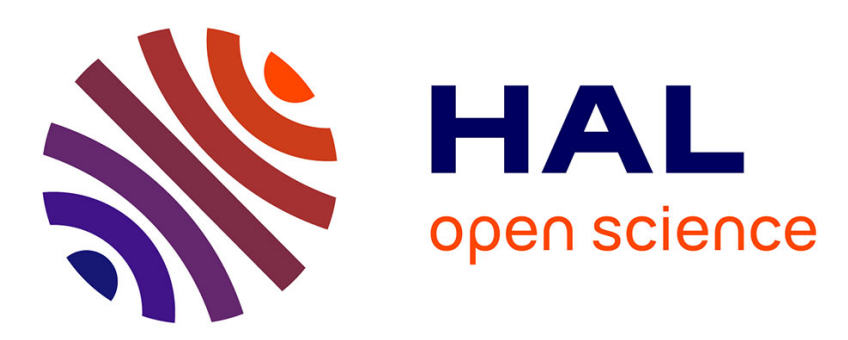

\title{
Phosphine-free palladium-catalysed direct C2-arylation of benzothiophenes with aryl bromides
}

\author{
Liqin Zhao, Christian Bruneau, Henri Doucet
}

\section{To cite this version:}

Liqin Zhao, Christian Bruneau, Henri Doucet. Phosphine-free palladium-catalysed direct C2arylation of benzothiophenes with aryl bromides. Tetrahedron, 2013, 69 (34), pp.7082-7089. 10.1016/j.tet.2013.06.037 . hal-00843040v2

\section{HAL Id: hal-00843040}

\section{https://hal-univ-rennes1.archives-ouvertes.fr/hal-00843040v2}

Submitted on 10 Jul 2013

HAL is a multi-disciplinary open access archive for the deposit and dissemination of scientific research documents, whether they are published or not. The documents may come from teaching and research institutions in France or abroad, or from public or private research centers.
L'archive ouverte pluridisciplinaire HAL, est destinée au dépôt et à la diffusion de documents scientifiques de niveau recherche, publiés ou non, émanant des établissements d'enseignement et de recherche français ou étrangers, des laboratoires publics ou privés. 
Phosphine-free palladium-catalyzed direct C2-arylation of benzothiophenes with aryl bromides Liqin Zhao, Christian Bruneau, Henri Doucet,* Institut des Sciences Chimiques de Rennes, UMR 6226 CNRS-Université de Rennes "Organométalliques: Matériaux et Catalyse", Campus de Beaulieu, 35042 Rennes, France.

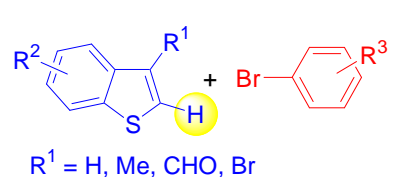

$\mathrm{R}^{1}=\mathrm{H}, \mathrm{Me}, \mathrm{CHO}, \mathrm{Br}$
$\mathrm{Pd}(\mathrm{OAc})_{2}$ 0.1-0.5 mol\% $\overrightarrow{D M F}$ or DMAc $\mathrm{KOAC}, 150^{\circ} \mathrm{C}$

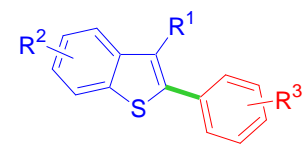




\title{
Phosphine-free palladium-catalyzed direct C2-arylation of benzothiophenes with aryl bromides
}

\author{
Liqin Zhao, Christian Bruneau, Henri Doucet* \\ Institut des Sciences Chimiques de Rennes, UMR 6226 CNRS-Université de Rennes "Organométalliques : Matériaux et \\ Catalyse", Campus de Beaulieu, 35042 Rennes, France. Fax: (+33)2-23-23-63-84 E-mail: henri.doucet@univ-rennes1.fr
}

\begin{abstract}
Ligand-free $\mathrm{Pd}(\mathrm{OAc})_{2}$ was found to catalyze very efficiently the direct $\mathrm{C} 2$-arylation of benzothiophene derivatives under low catalyst concentration. The reaction can be performed employing as little as $0.5-0.1$ mol- $\%$ catalyst with electron-deficient and some electron-rich aryl bromides. The presence of a methyl or a formyl substituent at $\mathrm{C} 3$ of benzothiophene has a minor influence on the reactivity, and even a bromo substituents at C3 is tolerated. A wide variety of functional groups on the aryl bromide such as nitrile, nitro, acetyl, formyl, ester, chloro, fluoro or trifluoromethyl has been employed. (C) 2013 Elsevier Science. All rights reserved
\end{abstract}

Keywords: palladium, catalysis, $\mathrm{C}-\mathrm{H}$ bond functionalization, benzothiophenes, aryl bromides

\section{Introduction}

Benzothiophene derivatives are of considerable interest for pharmaceutical chemistry due to their biological activities. For example, the 2-arylbenzothiophene derivative Raloxifene is used in the prevention of osteoporosis (Figure 1). Therefore, the development of simple and convenient processes using readily accessible benzothiophene derivatives for the synthesis of arylated benzothiophenes is highly desirable.

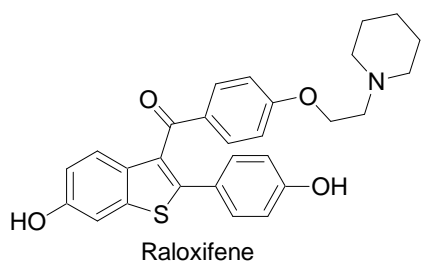

Figure 1. Example of bioactive 2-arylbenzothiophene.

The palladium-catalyzed direct arylation of several heteroaromatics such as furans, thiophenes or pyrroles via a $\mathrm{C}-\mathrm{H}$ bond activation using aryl halides has led to successes in recent years. ${ }^{1-5}$ Such couplings are very attractive compared to classical palladium-catalysed reactions such as Stille, Suzuki or Negishi couplings ${ }^{6}$ as they do not require the preliminary synthesis of organometallic derivatives. Some examples of such direct arylations using benzothiophenes have also been reported. ${ }^{7,8}$ However, the reported procedures either require $1-10$ mol- $\%$ palladium catalyst associated to $1-20$ mol- $\%$ of phosphine ligands ${ }^{[7]}$ or employed a stoichiometric amount of quaternary ammonium salts or crown ethers as stabilizing agents producing important amount of wastes. ${ }^{8}$ To our knowledge, the palladium-catalysed direct arylation of benzothiophenes using ligand-free catalyst without stabilizing agent have not been reported to date. The use of such conditions would be very attractive for industrial application, as it would reduce both the cost and wastes formation of such couplings.

In 2003, de Vries and co-workers have described extremely exciting results for the Heck and Suzuki reactions using a low loading (0.1-0.01 mol-\%) of ligand-free catalyst $\mathrm{Pd}(\mathrm{OAc})_{2}{ }^{9}{ }^{9}$ They have demonstrated that, at elevated temperature, when $\mathrm{Pd}(\mathrm{OAc})_{2}$ is employed as the catalyst precursor, soluble palladium(0) colloids or nanoparticles are formed, and that the Heck or Suzuki reaction takes place. We have recently reported that the use of the "de Vries conditions" allows the coupling or several heteroaromatics such as pyrroles, ${ }^{10 \mathrm{c}}$ imidazoles, ${ }^{10 \mathrm{~d}}$ furans, ${ }^{10 \mathrm{e}}$ thiophenes ${ }^{10 \mathrm{e}}$ or imidazopyridines $^{10 \mathrm{f}}$ using a very low loading (0.1-0.01 mol- $\%$ ) of ligand-free $\mathrm{Pd}(\mathrm{OAc})_{2}$ catalyst. ${ }^{10}$ However, so far, such procedure has not been employed for the direct arylation of benzothiophenes. ${ }^{11}$

Here, we wish to report on the coupling of benzothiophene derivatives with a variety of electronically and sterically diverse aryl and heteroaryl bromides using low loadings of ligand-free $\mathrm{Pd}(\mathrm{OAc})_{2}$ catalyst.

\section{Results and discussion}

First, we examined the influence of the reaction conditions for the coupling of benzothiophene with 4bromobenzonitrile (Scheme 1, Table 1). Starting form a slight excess of benzothiophene (1.5 eq.) with respect to the aryl bromide, in the presence of 0.5 mol- $\% \mathrm{Pd}(\mathrm{OAc})_{2}$ as the catalyst, KOAc as the base, and DMAc as the solvent at $150{ }^{\circ} \mathrm{C}$, the desired product 1 was obtained in $69 \%$ yield; whereas, a lower catalyst loading of 0.1 mol- $\%$ gave 1 in only $55 \%$ yield due to an uncomplete conversion of the aryl 
bromide (Table 1, entries 1 and 2). The influence of a few other bases was also examined; however, $\mathrm{NaOAc}, \mathrm{CsOAc}$ KF or carbonates led to lower yields of $\mathbf{1}$ (Table 1, entries 3-8). Then, we explored the influence of a few other solvents. Moderated yields were obtained in diethyl carbonate or cyclopentyl methyl ether (Table 1, entries 11 and 12). On the other hand, the use of only $0.1 \mathrm{~mol}-\%$ $\mathrm{Pd}(\mathrm{OAc})_{2}$ in DMF gave 1 in $59 \%$ yield (Table 1, entry 9).

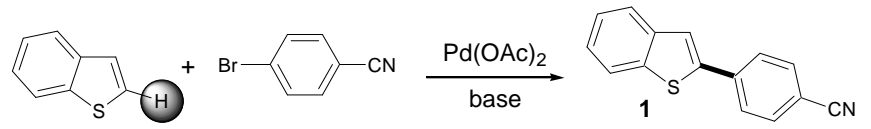

Scheme 1. Palladium-catalysed direct C2-arylation of benzothiophene with 4-bromobenzonitrile.

Table 1. Influence of the reaction conditions for palladiumcatalysed direct C2-arylation of benzothiophene with 4bromobenzonitrile (Scheme 1).

\begin{tabular}{|c|c|c|c|c|c|}
\hline Entry & $\begin{array}{c}\mathrm{Pd}(\mathrm{OAc})_{2} \\
(\mathrm{~mol}-\%)\end{array}$ & Solvent & Base & Conv. (\%) & $\begin{array}{l}\text { Yield in } \\
1(\%)\end{array}$ \\
\hline 1 & 0.5 & DMAc & KOAc & 100 & 69 \\
\hline 2 & 0.1 & DMAc & KOAc & 67 & 55 \\
\hline 3 & 0.1 & DMAc & $\mathrm{CsOAc}$ & 12 & - \\
\hline 4 & 0.1 & DMAc & $\mathrm{NaOAc}$ & 43 & 37 \\
\hline 5 & 0.1 & DMAc & $\mathrm{Na}_{2} \mathrm{CO}_{3}$ & 25 & 18 \\
\hline 6 & 0.1 & DMAc & $\mathrm{K}_{2} \mathrm{CO}_{3}$ & 7 & 6 \\
\hline 7 & 0.1 & DMAc & $\mathrm{Cs}_{2} \mathrm{CO}_{3}$ & 90 & $0^{\mathrm{a}}$ \\
\hline 8 & 0.1 & DMAc & KF & 36 & 30 \\
\hline 9 & 0.1 & $\mathrm{DMF}$ & KOAc & 80 & $59^{\mathrm{b}}$ \\
\hline 10 & 0.5 & NMP & KOAc & 70 & $55^{\mathrm{b}}$ \\
\hline 11 & 0.5 & diethyl carbonate & KOAc & 54 & $48^{\mathrm{b}}$ \\
\hline 12 & 0.5 & $\begin{array}{c}\text { cyclopentyl methyl } \\
\text { ether }\end{array}$ & KOAc & 51 & $42^{\mathrm{b}}$ \\
\hline 13 & 0.5 & pentan-1-ol & KOAc & 10 & $-{ }^{b}$ \\
\hline
\end{tabular}
$\mathrm{mmol})$, base $(2 \mathrm{mmol}), 150^{\circ} \mathrm{C}, 16 \mathrm{~h}$, under argon, conversion of $4-$ bromobenzonitrile. ${ }^{a}$ Formation of 4 -bromobenzamide. ${ }^{b}$ Catalyst is a solution prepared with $1 \mathrm{mg}$ of $\mathrm{Pd}(\mathrm{OAc})_{2}$ in $1 \mathrm{~mL}$ of DMAc.

We then studied the scope of 2-arylation of benzothiophene using 0.1 or 0.5 mol- $\% \mathrm{Pd}(\mathrm{OAc})_{2}$ catalyst, KOAc as the base and either DMF or DMAc as the solvent (Scheme 2, Table 2). A good yield in 4 was obtained from 4bromobenzaldehyde using only $0.1 \mathrm{~mol}-\% \mathrm{Pd}(\mathrm{OAc})_{2}$ (Table 2, entry 4). However, in most cases, 0.5 mol-\% $\mathrm{Pd}(\mathrm{OAc})_{2}$ had to be employed to observe high conversions of the aryl bromides and good yields of coupling products. For example, with this catalyst loading, 4trifluoromethylbromobenzene, 4-bromoacetophenone, ethyl 4-bromobenzoate, 4-chlorobromobenzene or 4fluorobromobenzene gave the expected $\mathrm{C} 2$-arylated benzothiophenes 3, 5-8 in 61-80\% yields (Table 2, entries 3, 512). With these reactants, very similar yields were obtained using DMF or DMAc as the solvent. It should be noted that no cleavage of the $\mathrm{C}-\mathrm{Cl}$ bond of 4chlorobromobenzene was observed under these reaction conditions allowing further transformations. In the presence of the electron-rich aryl bromides, 4-bromotoluene and 4-tert-butylbromobenzene, moderate yields in $\mathbf{9}$ and $\mathbf{1 0}$ were obtained, due to partial conversion of these aryl bro- mides (Table 2, entries 13 and 14).

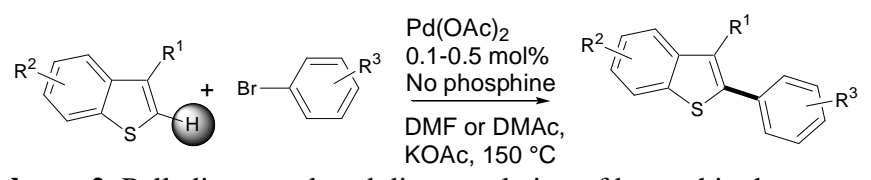

Scheme 2. Palladium-catalysed direct arylation of benzothiophene derivatives with (hetero)aryl bromides.

Table 2. Palladium-catalysed arylation of benzothiophene with (hetero)aryl bromides (Scheme 2).

(mol-\%)

Conditions: Catalyst is a solution prepared with $1 \mathrm{mg}$ of $\mathrm{Pd}(\mathrm{OAc})_{2}$ in $1 \mathrm{~mL}$ of DMAc (0.001 or 0.005 eq.), benzothiophene (1.5 eq.), 
aryl bromide (1 eq.), KOAc (2 eq.), DMF, $150{ }^{\circ} \mathrm{C}, 16 \mathrm{~h}$, isolated yields. ${ }^{a}$ Solvent: DMAc.

With such substrates, the use of palladium associated to phosphine ligands should be preferred. A few meta- and ortho-substituted aryl bromides were also employed. 2and 3-bromobenzonitriles gave 11 and 14 in $60 \%$ and $73 \%$ yields, respectively in the presence of $0.1 \mathrm{~mol}-\% \mathrm{Pd}(\mathrm{OAc})_{2}$ (Table 2, entries 15 and 18). Both 1- and 2bromonaphthalene gave the expected products $\mathbf{1 5}$ and $\mathbf{1 3}$ (Table 2, entries 17 and 19). However, from 1bromonaphthalene, a higher yield in $\mathbf{1 5}$ was obtained when using DMAc as the solvent. A similar tendency was observed with 9-bromoanthracene. In DMF, a very low yield in 16 was observed by GC/MS analysis due to the formation of several unidentified side-products; whereas a very clean reaction was observed in DMAc to give $\mathbf{1 6}$ in $83 \%$ yield (Table 2, entries 20 and 21). Therefore, for challenging aryl bromides, the use of DMAc as the solvent should be preferred. We also examined the coupling of 5bromopyrimidine with benzothiophene. The desired product 22 was isolated in $66 \%$ yield (Table 2, entry 22). For all these reactions, no arylation at $\mathrm{C} 3$ of benzothiophene was detected.

We then studied the reactivity of benzothiophene-3carbaldehyde with some aryl bromides (Table 3). We observed that using $0.1 \mathrm{~mol}-\% \mathrm{Pd}(\mathrm{OAc})_{2}$ as the catalyst and 4-bromobenzonitrile as coupling partner in DMF, the 2arylated benzothiophene $\mathbf{1 8}$ was obtained in low yield (Table 3, entry 1). Moreover, the formation of the homocoupling product biphenyl-4,4'-dicarbonitrile was also observed.

Table 3. Palladium-catalysed C2-arylation of benzothiophene-3carbaldehyde with (hetero)aryl bromides (Scheme 2).

Entry Aryl halide

Conditions: benzothiophene-3-carbaldehyde (1.5 eq.), aryl bromide (1 eq.), KOAc (2 eq.), DMAc, $150{ }^{\circ} \mathrm{C}, 16 \mathrm{~h}$, isolated yields.

${ }^{\text {a }}$ In DMF with a solution of catalyst prepared with $1 \mathrm{mg}$ of $\mathrm{Pd}(\mathrm{OAc})_{2}$ in $1 \mathrm{~mL}$ of DMAc.
The use of a higher catalyst loading allowed to improve the yield in 18 to $33 \%$, but some formation of unidentified side-products was observed, and again, some formation of biphenyl-4,4'-dicarbonitrile was detected (Table 3, entry 2). On the other hand, in DMAc, using 0.5 mol-\% $\mathrm{Pd}(\mathrm{OAc})_{2}$, a clean reaction was observed, and $\mathbf{1 8}$ was isolated in $65 \%$ yield (Table 3, entry 3 ). The stability of benzothiophene-3carbaldehyde in DMF appears to be limited. From the more congested substrate 2-bromobenzonitrile, a good yield in $\mathbf{2 0}$ was also obtained in DMAc (Table 3, entry 5). A similar reactivity of 3-bromonitrobenzene, 4bromoisoquinoline or 5-bromopyrimidine was observed in DMAc to give 19, 21 and 22 in $61-78 \%$ yields (Table 2, entries 4, 6 and 7).

With 3-methylbenzothiophene in DMAc, very clean reactions were observed in all cases using various aryl bromides and 0.1-0.5 mol-\% $\mathrm{Pd}(\mathrm{OAc})_{2}$ catalyst (Table 4). The electron-deficient aryl bromides, 4-bromobenzonitrile and ethyl 4-bromobenzoate gave $\mathbf{2 3}$ and $\mathbf{2 4}$ in $88 \%$ and $81 \%$ yields, respectively using $0.5 \mathrm{~mol}-\% \mathrm{Pd}(\mathrm{OAc})_{2}$ catalyst (Table 4, entries 1 and 2). A high yield in 25 resulting from selective cleavage of the $\mathrm{C}$-Br bond was also obtained from 4-chlorobromobenzene (Table 4, entries 3 and 4). The use of only 0.1 mol- $\% \mathrm{Pd}(\mathrm{OAc})_{2}$ led to a high conversion of this aryl bromide.

Table 4. Palladium-catalysed C2-arylation of 3methylbenzothiophene with (hetero)aryl bromides (Scheme 2).

\begin{tabular}{|c|c|c|c|}
\hline Entry & Aryl halide & Product & $\begin{array}{l}\text { Catalyst Yield } \\
(\mathrm{mol}-\%)(\%)\end{array}$ \\
\hline
\end{tabular}

(1)

Conditions: $\quad \operatorname{Pd}(\mathrm{OAc})_{2} \quad(0.001 \quad$ or $0.005 \quad$ eq. $)$, 3methylbenzothiophene (1.5 eq.), aryl bromide (1 eq.), KOAc (2 eq.), DMAc, $150{ }^{\circ} \mathrm{C}, 16 \mathrm{~h}$, isolated yields. 
Again, no cleavage of the $\mathrm{C}-\mathrm{Cl}$ bond was observed allowing further transformations. A slightly lower yield was obtained for the coupling of the electron-rich aryl bromide: 3-bromotoluene (Table 4, entry 5). It should be noted that both 3-bromopyridine and 4-bromoisoquinoline could be coupled with 3-methylbenzothiophene to give $\mathbf{2 8}$ and $\mathbf{2 9}$ in high yields using only 0.1 mol-\% $\mathrm{Pd}(\mathrm{OAc})_{2}$ (Table 4 , entries 7 and 8).

The reactivity of 5-chloro-3-methylbenzothiophene with a set of (hetero)aryl bromides was also examined using 0.10.5 mol-\% $\mathrm{Pd}(\mathrm{OAc})_{2}$ catalyst (Table 5). In all cases, high yields of the desired coupling products 30-38 were obtained, except with the electron-rich, 4-tertbutylbromobenzene. For example, from 4chlorobromobenzene, 3-nitrobromobenzene or 2bromobenzonitrile and $0.1 \mathrm{~mol}-\% \mathrm{Pd}(\mathrm{OAc})_{2}$, the products 32, 34 and 37 were obtained in $82-85 \%$ yields (Table 5, entries 3, 5 and 8).

Table 5. Palladium-catalysed C2-arylation of 5-chloro-3methylbenzothiophene with (hetero)aryl bromides (Scheme 2).

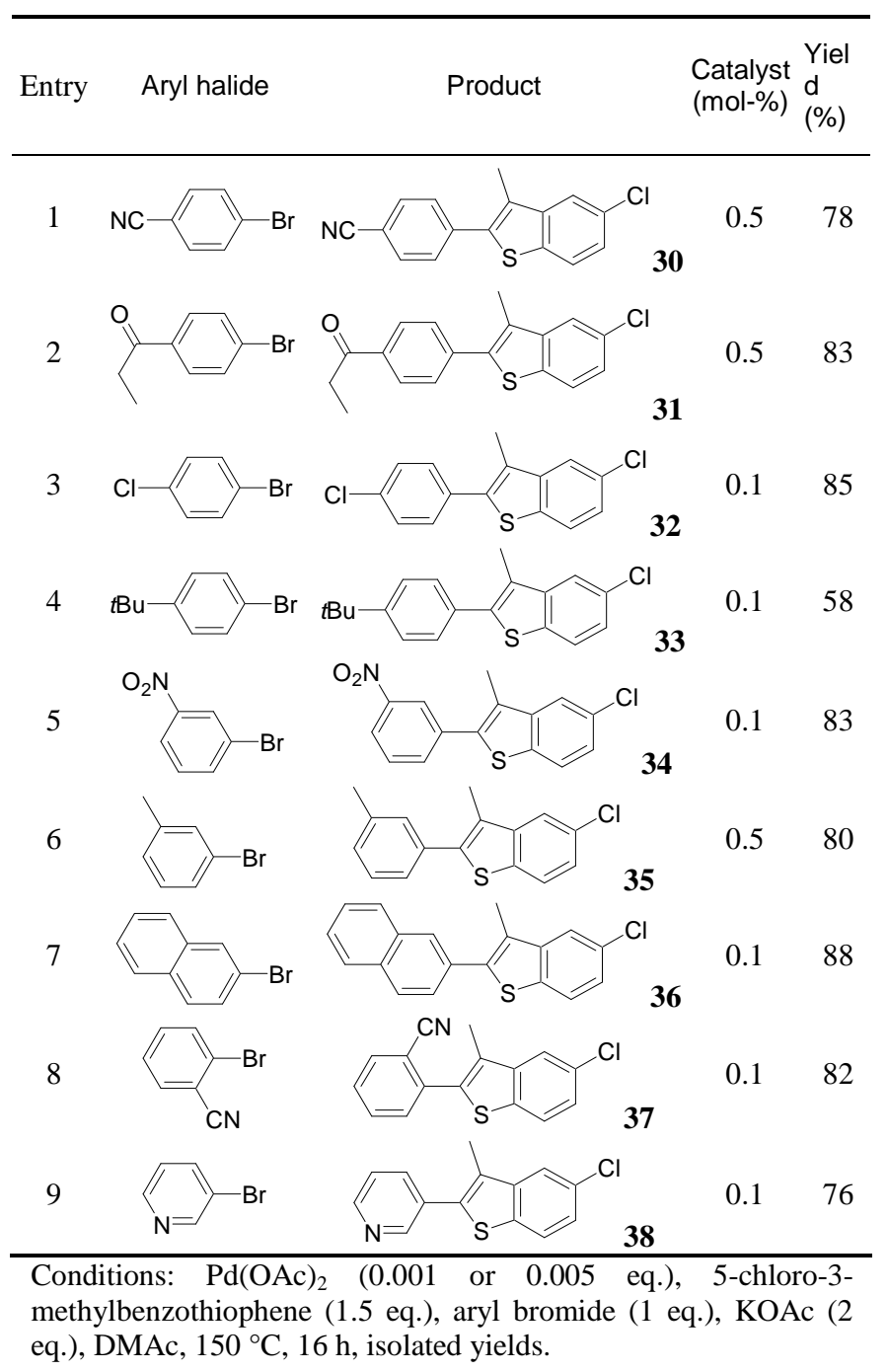

In order to have a better insight of the influence of the benzothiophenes $\mathrm{C} 3$-substituents on their reactivity, we performed a competitive experiment using an equimolar mixture of 3-methylbenzothiophene and benzothiophene-3carbaldehyde in the presence of 4-bromobenzonitrile and 0.5 mol-\% $\mathrm{Pd}(\mathrm{OAc})_{2}$ (Scheme 3 ). We observed the formation of a mixture of the products $\mathbf{2 3}$ and $\mathbf{1 8}$ in a 41:59 ratio. This result indicates that the presence of electron-donating or electron-withdrawing substituents at C3 of benzothiophenes only has a minor influence on their reactivity.
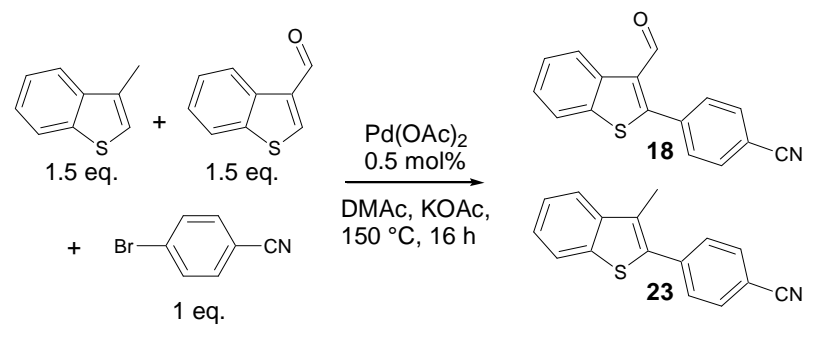

Ratio 18:23 = 59:41

Scheme 3. Competitive experiments using a mixture of 3methylbenzothiophene and benzothiophene-3-carbaldehyde.

To our knowledge, the direct arylation at $\mathrm{C} 2$ of 3bromobenzothiophene has not been reported. So far, the coupling of such reactants to prepare 3arylbenzothiophenes employs organozinc intermediates. ${ }^{12}$ We observed that using 3-bromobenzothiophene and 2bromobenzonitrile in a 1.5:1 ratio and again 0.5 mol-\% $\mathrm{Pd}(\mathrm{OAc})_{2}$ catalyst, the desired product 39 was obtained in $62 \%$ yield without cleavage of the $\mathrm{C}-\mathrm{Br}$ bond of the benzothiophene derivative.

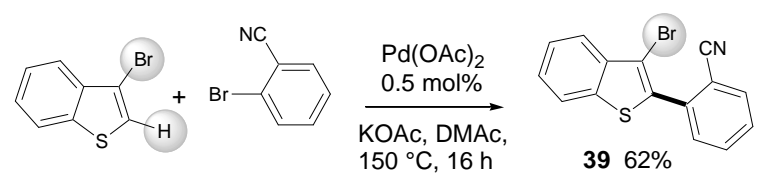

Scheme 4. Palladium-catalysed direct arylation of 3bromobenzothiophene with 2-bromobenzonitrile.

Finally, the palladium-catalysed C3 direct arylation of 7 was examined (Scheme 5). The use of 0.5 mol-\% $\mathrm{Pd}(\mathrm{OAc})_{2}$ catalyst gave $\mathbf{4 0}$ as traces. On the other hand, in the presence of $0.5 \mathrm{~mol}-\% \mathrm{PdCl}\left(\mathrm{C}_{3} \mathrm{H}_{5}\right)(\mathrm{dppb})$ catalyst, 40 was isolated in $47 \%$ yield, without cleavage of the $\mathrm{C}-\mathrm{Cl}$ bond of the aryl substituent, showing the beneficial effect of the phosphine ligand in this case.

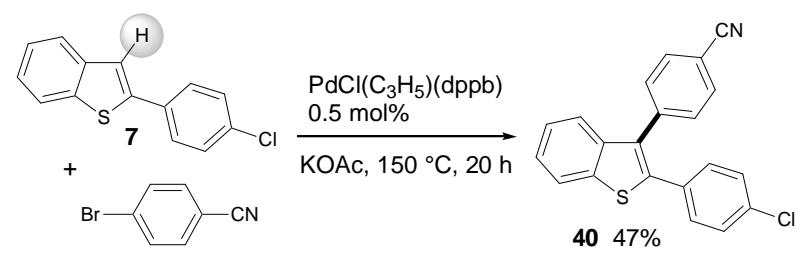

Scheme 5. Palladium-catalysed direct C3 arylation of 2arylbenzothiophene $\mathbf{7}$ with 4-bromobenzonitrile. 
In summary, we have demonstrated that using as little as $0.5-0.1$ mol- $\%$ of $\mathrm{Pd}(\mathrm{OAc})_{2}$ as the catalyst precursor, the direct 2-arylation via $\mathrm{C}-\mathrm{H}$ bond activation of benzothiophenes proceeds in moderate to high yields. This procedure gave the best results using electron-deficient aryl bromides. Several functions such as formyl, acetyl, propionyl, nitro, nitrile, chloro, fluoro or trifluoromethyl are tolerated. Congested aryl bromides such as 9-bromoanthracene, also gave satisfactory results. This ligand-free low catalyst loading procedure is economically and environmentally attractive, as there is no need to eliminate phosphine derivatives at the end of the reaction; and as with this $\mathrm{C}-\mathrm{H}$ bond activation procedure, no preparation of an organometallic derivative is required, reducing the number of steps and therefore the mass of waste products. The major waste is the relatively non-toxic $\mathrm{AcOH} / \mathrm{KBr}$ instead of metallic salts with more classical metal-catalysed coupling reactions. For these reasons, the methodology developed here is very promising for the sustainable synthesis of 2arylbenzothiophenes.

\section{Experimental}

General: All reactions were performed in Schlenck tubes under argon. Potassium acetate 99+ was used. Benzothiophenes and aryl bromides were used without purification. ${ }^{1} \mathrm{H}(400 \mathrm{MHz}, 25$ $\left.{ }^{\circ} \mathrm{C}\right),{ }^{13} \mathrm{C}\left(100 \mathrm{MHz}, 25{ }^{\circ} \mathrm{C}\right)$ spectra were recorded in $\mathrm{CDCl}_{3}$ solutions. Chemical shifts are reported in ppm relative to $\mathrm{CDCl}_{3}\left({ }^{1} \mathrm{H}\right.$ : 7.29 and ${ }^{13} \mathrm{C}$ : 77.0). Flash chromatography was performed on silica gel (230-400 mesh).

\section{General procedure for the preparation of 1-40:}

As a typical experiment, the reaction of the aryl bromide (1 mmol), benzothiophene $(1.5 \mathrm{mmol})$ and KOAc $(0.196 \mathrm{~g}, 2 \mathrm{mmol})$ at $150{ }^{\circ} \mathrm{C}$ during $16 \mathrm{~h}$ in DMF or DMAc $(4 \mathrm{~mL})$ in the presence of $\mathrm{Pd}(\mathrm{OAc})_{2}(0.224 \mathrm{mg}, 0.001 \mathrm{mmol})$ or $(1.12 \mathrm{mg}, 0.005 \mathrm{mmol})$ prepared as a solution in DMAc $\left(1 \mathrm{mg}\right.$ of $\mathrm{Pd}(\mathrm{OAc})_{2}$ in $1 \mathrm{~mL}$ of DMAc) under argon affords the coupling product after evaporation of the solvent and purification on silica gel.

\section{2-(4-Cyanophenyl)-benzo[b]thiophene (1)}

From 4-bromobenzonitrile $(0.182 \mathrm{~g}, 1 \mathrm{mmol})$ and benzothiophene $(0.201 \mathrm{~g}, 1.5 \mathrm{mmol}), 1$ was obtained in $69 \%(0.162 \mathrm{~g})$ yield. ${ }^{1} \mathrm{H}$ NMR $\left(400 \mathrm{MHz}, \mathrm{CDCl}_{3}\right): \square \delta 7.76(\mathrm{~d}, J=8.1 \mathrm{~Hz}, 1 \mathrm{H}), 7.72(\mathrm{~d}, J$ $=7.9 \mathrm{~Hz}, 1 \mathrm{H}), 7.68(\mathrm{~d}, J=8.4 \mathrm{~Hz}, 2 \mathrm{H}), 7.59(\mathrm{~d}, J=8.4 \mathrm{~Hz}, 2 \mathrm{H})$, $7.55(\mathrm{~s}, 1 \mathrm{H}), 7.30(\mathrm{t}, J=7.3 \mathrm{~Hz}, 1 \mathrm{H}), 7.28(\mathrm{t}, J=7.3 \mathrm{~Hz}, 1 \mathrm{H})$.

\section{2-(4-Nitrophenyl)-benzo[b]thiophene (2)}

From 4-bromonitrobenzene $(0.202 \mathrm{~g}, 1 \mathrm{mmol})$ and benzothiophene $(0.201 \mathrm{~g}, 1.5 \mathrm{mmol}), 2$ was obtained in $51 \%$ $(0.130 \mathrm{~g})$ yield. ${ }^{1} \mathrm{H}$ NMR $\left(400 \mathrm{MHz}, \mathrm{CDCl}_{3}\right):{ }^{\prime} \delta 8.20(\mathrm{~d}, J=8.4$ $\mathrm{Hz}, 2 \mathrm{H}), 7.82-7.70(\mathrm{~m}, 4 \mathrm{H}), 7.64(\mathrm{~s}, 1 \mathrm{H}), 7.33(\mathrm{t}, J=7.3 \mathrm{~Hz}, 1 \mathrm{H})$, $7.31(\mathrm{t}, J=7.3 \mathrm{~Hz}, 1 \mathrm{H})$.

\section{2-[4-(Trifluoromethyl)phenyl]-benzo[b]thiophene $(3)^{7 \mathrm{j}}$}

From 1-bromo-4-trifluoromethylbenzene $(0.225 \mathrm{~g}, 1 \mathrm{mmol})$ and benzothiophene $(0.201 \mathrm{~g}, 1.5 \mathrm{mmol}), 3$ was obtained in $61 \%$ $(0.170 \mathrm{~g})$ yield. ${ }^{1} \mathrm{H}$ NMR $\left(400 \mathrm{MHz}, \mathrm{CDCl}_{3}\right): \cdot \delta$ 7.80-7.70 (m, $4 \mathrm{H}), 7.61(\mathrm{~d}, J=8.2 \mathrm{~Hz}, 2 \mathrm{H}), 7.56(\mathrm{~s}, 1 \mathrm{H}), 7.32(\mathrm{t}, J=7.3 \mathrm{~Hz}$, $1 \mathrm{H}), 7.28(\mathrm{t}, J=7.3 \mathrm{~Hz}, 1 \mathrm{H})$.
4-Benzo[b]thiophen-2-ylbenzaldehyde $(4)^{1}$

From 4-bromobenzaldehyde $(0.185 \mathrm{~g}, 1 \mathrm{mmol})$ and benzothiophene $(0.201 \mathrm{~g}, 1.5 \mathrm{mmol}), 4$ was obtained in $72 \%$ $(0.171 \mathrm{~g})$ yield. ${ }^{1} \mathrm{H}$ NMR $\left(400 \mathrm{MHz}, \mathrm{CDCl}_{3}\right):{ }^{*} \quad \delta .97(\mathrm{~s}, 1 \mathrm{H})$, $7.87(\mathrm{~d}, J=8.3 \mathrm{~Hz}, 2 \mathrm{H}), 7.80(\mathrm{~d}, J=8.3 \mathrm{~Hz}, 2 \mathrm{H}), 7.80-7.72(\mathrm{~m}$, $2 \mathrm{H}), 7.63(\mathrm{~s}, 1 \mathrm{H}), 7.32(\mathrm{t}, J=7.3 \mathrm{~Hz}, 1 \mathrm{H}), 7.28(\mathrm{t}, J=7.3 \mathrm{~Hz}$, $1 \mathrm{H})$.

\section{1-(4-Benzo[b]thiophen-2-yl-phenyl)-ethanone $(5)^{1}$}

From 4-bromoacetophenone $(0.199 \mathrm{~g}, \quad 1 \mathrm{mmol})$ and benzothiophene $(0.201 \mathrm{~g}, 1.5 \mathrm{mmol}), 5$ was obtained in $66 \%$ $(0.166 \mathrm{~g})$ yield. ${ }^{1} \mathrm{H} \mathrm{NMR}\left(400 \mathrm{MHz}, \mathrm{CDCl}_{3}\right):=\delta 7.93(\mathrm{~d}, J=8.3$ $\mathrm{Hz}, 2 \mathrm{H}), 7.80-7.68(\mathrm{~m}, 4 \mathrm{H}), 7.59(\mathrm{~s}, 1 \mathrm{H}), 7.30$ (t, $J=7.3 \mathrm{~Hz}, 1 \mathrm{H})$, $7.27(\mathrm{t}, J=7.3 \mathrm{~Hz}, 1 \mathrm{H}), 2.55(\mathrm{~s}, 3 \mathrm{H})$.

\section{Ethyl 4-benzo[b]thiophen-2-yl-benzoate $(6)^{\text {th }}$}

From ethyl 4-bromobenzoate $(0.229 \mathrm{~g}, \quad 1 \mathrm{mmol})$ and benzothiophene $(0.201 \mathrm{~g}, 1.5 \mathrm{mmol}), 6$ was obtained in $76 \%$ $(0.214 \mathrm{~g})$ yield. ${ }^{1} \mathrm{H}$ NMR $\left(400 \mathrm{MHz}, \mathrm{CDCl}_{3}\right):{ }^{*} \delta 8.01(\mathrm{~d}, J=8.3$ $\mathrm{Hz}, 2 \mathrm{H}), 7.74(\mathrm{~d}, J=7.0 \mathrm{~Hz}, 1 \mathrm{H}), 7.70-7.65(\mathrm{~m}, 3 \mathrm{H}), 7.56(\mathrm{~s}, 1 \mathrm{H})$, $7.30(\mathrm{t}, J=7.3 \mathrm{~Hz}, 1 \mathrm{H}), 7.27(\mathrm{t}, J=7.3 \mathrm{~Hz}, 1 \mathrm{H}), 4.32(\mathrm{q}, J=7.2$ $\mathrm{Hz}, 2 \mathrm{H}), 1.34(\mathrm{t}, J=7.2 \mathrm{~Hz}, 3 \mathrm{H})$.

\section{2-(4-Chlorophenyl)-benzo[b]thiophene (7) ${ }^{7 \mathrm{~h}}$}

From 1-bromo-4-chlorobenzene $(0.191 \mathrm{~g}, 1 \mathrm{mmol})$ and benzothiophene $(0.201 \mathrm{~g}, 1.5 \mathrm{mmol}), 7$ was obtained in $73 \%$ $(0.178 \mathrm{~g})$ yield. ${ }^{1} \mathrm{H}$ NMR $\left(400 \mathrm{MHz}, \mathrm{CDCl}_{3}\right): \cdot \delta 7.76(\mathrm{~d}, J=7.0$ $\mathrm{Hz}, 1 \mathrm{H}), 7.70(\mathrm{~d}, J=7.0 \mathrm{~Hz}, 1 \mathrm{H}), 7.56(\mathrm{~d}, J=8.3 \mathrm{~Hz}, 2 \mathrm{H}), 7.44$ (s, 1H), $7.32(\mathrm{~d}, J=8.3 \mathrm{~Hz}, 2 \mathrm{H}), 7.30-7.20(\mathrm{~m}, 2 \mathrm{H})$.

\section{2-(4-Fluorophenyl)-benzo[b]thiophene $(8)^{7 \mathrm{~h}}$}

From 1-bromo-4-fluorobenzene $(0.175 \mathrm{~g}, 1 \mathrm{mmol})$ and benzothiophene $(0.201 \mathrm{~g}, 1.5 \mathrm{mmol}), \mathbf{8}$ was obtained in $80 \%$ $(0.182 \mathrm{~g})$ yield. ${ }^{1} \mathrm{H}$ NMR $\left(400 \mathrm{MHz}, \mathrm{CDCl}_{3}\right): \cdot \delta 7.73(\mathrm{~d}, J=7.7$ $\mathrm{Hz}, 1 \mathrm{H}), 7.68(\mathrm{~d}, J=7.7 \mathrm{~Hz}, 1 \mathrm{H}), 7.58(\mathrm{dd}, J=8.6,4.8 \mathrm{~Hz}, 2 \mathrm{H})$, $7.37(\mathrm{~s}, 1 \mathrm{H}), 7.27(\mathrm{t}, J=7.3 \mathrm{~Hz}, 1 \mathrm{H}), 7.23$ (t, $J=7.3 \mathrm{~Hz}, 1 \mathrm{H})$, $7.03(\mathrm{t}, J=8.6 \mathrm{~Hz}, 2 \mathrm{H})$.

\section{2-(4-Methylphenyl)-benzo[b]thiophene $(9)^{71}$}

From 4-bromotoluene $(0.171 \mathrm{~g}, 1 \mathrm{mmol})$ and benzothiophene $(0.201 \mathrm{~g}, 1.5 \mathrm{mmol}), 9$ was obtained in $55 \%(0.123 \mathrm{~g})$ yield. ${ }^{1} \mathrm{H}$ $\operatorname{NMR}\left(400 \mathrm{MHz}, \mathrm{CDCl}_{3}\right):{ }^{\prime} \delta 7.74(\mathrm{~d}, J=7.8 \mathrm{~Hz}, 1 \mathrm{H}), 7.68(\mathrm{~d}, J$ $=7.8 \mathrm{~Hz}, 1 \mathrm{H}), 7.53(\mathrm{~d}, J=8.3 \mathrm{~Hz}, 2 \mathrm{H}), 7.42(\mathrm{~s}, 1 \mathrm{H}), 7.26(\mathrm{t}, J=$ $7.3 \mathrm{~Hz}, 1 \mathrm{H}), 7.23(\mathrm{t}, J=7.3 \mathrm{~Hz}, 1 \mathrm{H}), 7.20(\mathrm{~d}, J=8.3 \mathrm{~Hz}, 2 \mathrm{H})$, $2.32(\mathrm{~s}, 3 \mathrm{H})$.

\section{2-(4-tert-Butylphenyl)-benzo[b]thiophene $(10)^{7 \mathrm{~h}}$}

From 1-tert-butylbromobenzene $(0.213 \mathrm{~g}, 1 \mathrm{mmol})$ and benzothiophene $(0.201 \mathrm{~g}, 1.5 \mathrm{mmol}), \mathbf{1 0}$ was obtained in $32 \%$ $(0.085 \mathrm{~g})$ yield. ${ }^{1} \mathrm{H}$ NMR $\left(400 \mathrm{MHz}, \mathrm{CDCl}_{3}\right):^{\cdot} \delta 7.73(\mathrm{~d}, J=7.8$ $\mathrm{Hz}, 1 \mathrm{H}), 7.67(\mathrm{~d}, J=7.8 \mathrm{~Hz}, 1 \mathrm{H}), 7.53(\mathrm{~d}, J=8.3 \mathrm{~Hz}, 2 \mathrm{H}), 7.42$ $(\mathrm{s}, 1 \mathrm{H}), 7.36(\mathrm{~d}, J=8.3 \mathrm{~Hz}, 2 \mathrm{H}), 7.24(\mathrm{t}, J=7.3 \mathrm{~Hz}, 1 \mathrm{H}), 7.21(\mathrm{t}$, $J=7.3 \mathrm{~Hz}, 1 \mathrm{H}), 1.27(\mathrm{~s}, 9 \mathrm{H})$.

\section{2-(3-Cyanophenyl)-benzo[b]thiophene (11) ${ }^{13 a}$}

From 3-bromobenzonitrile $(0.182 \mathrm{~g}, 1 \mathrm{mmol})$ and benzothiophene $(0.201 \mathrm{~g}, 1.5 \mathrm{mmol}), 11$ was obtained in $60 \%(0.141 \mathrm{~g})$ yield. ${ }^{1} \mathrm{H}$ NMR $\left(400 \mathrm{MHz}, \mathrm{CDCl}_{3}\right)$ : $\quad \delta 7.88(\mathrm{~s}, 1 \mathrm{H}), 7.81(\mathrm{~d}, J=7.9 \mathrm{~Hz}$, $1 \mathrm{H}), 7.76(\mathrm{~d}, J=7.8 \mathrm{~Hz}, 1 \mathrm{H}), 7.71(\mathrm{~d}, J=7.8 \mathrm{~Hz}, 1 \mathrm{H}), 7.51(\mathrm{~d}, J$ $=7.9 \mathrm{~Hz}, 1 \mathrm{H}), 7.50(\mathrm{~s}, 1 \mathrm{H}), 7.43(\mathrm{t}, J=7.8 \mathrm{~Hz}, 1 \mathrm{H}), 7.30(\mathrm{t}, J=$ $7.3 \mathrm{~Hz}, 1 \mathrm{H}), 7.27(\mathrm{t}, J=7.3 \mathrm{~Hz}, 1 \mathrm{H})$.

\section{2-m-Tolylbenzo[b]thiophene (12) ${ }^{13 b}$}

From 3-bromotoluene $(0.171 \mathrm{~g}, 1 \mathrm{mmol})$ and benzothiophene $(0.201 \mathrm{~g}, 1.5 \mathrm{mmol}), 12$ was obtained in $60 \%(0.134 \mathrm{~g})$ yield. ${ }^{1} \mathrm{H}$ 
$\operatorname{NMR}\left(400 \mathrm{MHz}, \mathrm{CDCl}_{3}\right): \square \delta 7.75(\mathrm{~d}, J=7.7 \mathrm{~Hz}, 1 \mathrm{H}), 7.69(\mathrm{~d}, J$ $=7.5 \mathrm{~Hz}, 1 \mathrm{H}), 7.50-7.40(\mathrm{~m}, 3 \mathrm{H}), 7.25-7.20(\mathrm{~m}, 3 \mathrm{H}), 7.08(\mathrm{~d}, J=$ $7.4 \mathrm{~Hz}, 1 \mathrm{H}), 2.34(\mathrm{~s}, 3 \mathrm{H})$.

\section{2-Naphthalen-2-yl-benzo[b]thiophene $(13)^{7 \mathrm{~h}}$}

From 2-bromonaphthalene $(0.207 \mathrm{~g}, 1 \mathrm{mmol})$ and benzothiophene $(0.201 \mathrm{~g}, 1.5 \mathrm{mmol}), \mathbf{1 3}$ was obtained in $62 \%(0.161 \mathrm{~g})$ yield. ${ }^{1} \mathrm{H}$ NMR $\left(400 \mathrm{MHz}, \mathrm{CDCl}_{3}\right): \square \delta 8.07(\mathrm{~s}, 1 \mathrm{H}), 7.85-7.70(\mathrm{~m}, 6 \mathrm{H})$, $7.60(\mathrm{~s}, 1 \mathrm{H}), 7.47-7.37(\mathrm{~m}, 2 \mathrm{H}), 7.29(\mathrm{t}, J=7.8 \mathrm{~Hz}, 1 \mathrm{H}), 7.23(\mathrm{t}, J$ $=7.3 \mathrm{~Hz}, 1 \mathrm{H})$

\section{2-Benzo[b]thiophen-2-yl-benzonitrile (14)}

From 2-bromobenzonitrile $(0.182 \mathrm{~g}, 1 \mathrm{mmol})$ and benzothiophene $(0.201 \mathrm{~g}, 1.5 \mathrm{mmol}), \mathbf{1 4}$ was obtained in $73 \%(0.171 \mathrm{~g})$ yield as a white solid (mp 119-121 $\left.{ }^{\circ} \mathrm{C}\right) .{ }^{1} \mathrm{H}$ NMR $\left(400 \mathrm{MHz}, \mathrm{CDCl}_{3}\right)$ : $\square \delta$ 7.80-7.72 (m, 3H), $7.67(\mathrm{~d}, J=7.7 \mathrm{~Hz}, 1 \mathrm{H}), 7.59(\mathrm{~d}, J=7.8 \mathrm{~Hz}$, $1 \mathrm{H}), 7.52(\mathrm{t}, J=7.3 \mathrm{~Hz}, 1 \mathrm{H}), 7.36-7.23(\mathrm{~m}, 3 \mathrm{H}) .{ }^{13} \mathrm{C}$ NMR $(100$ $\left.\mathrm{MHz}, \mathrm{CDCl}_{3}\right): \delta 140.3,140.2,139.2,137.5,134.5,133.0,130.2$, 128.2, 125.3, 124.8, 124.5, 124.4, 122.1, 118.7, 110.5. elemental analysis: calcd (\%) for $\mathrm{C}_{15} \mathrm{H}_{9} \mathrm{NS}$ (235.30): C 76.56, H 3.86; found: C 76.65, H 4.02 .

\section{2-Naphthalen-1-ylbenzothiophene (15) ${ }^{71}$}

From 1-bromonaphthalene $(0.207 \mathrm{~g}, 1 \mathrm{mmol})$ and benzothiophene $(0.201 \mathrm{~g}, 1.5 \mathrm{mmol}), \mathbf{1 5}$ was obtained in $82 \%(0.213 \mathrm{~g})$ yield. ${ }^{1} \mathrm{H}$ NMR $\left(400 \mathrm{MHz}, \mathrm{CDCl}_{3}\right): \square \delta 8.18(\mathrm{~d}, J=8.0 \mathrm{~Hz}, 1 \mathrm{H}), 7.83-7.75$ $(\mathrm{m}, 3 \mathrm{H}), 7.73(\mathrm{~d}, J=7.6 \mathrm{~Hz}, 1 \mathrm{H}), 7.54(\mathrm{~d}, J=7.0 \mathrm{~Hz}, 1 \mathrm{H}), 7.45-$ $7.22(\mathrm{~m}, 6 \mathrm{H})$.

\section{2-Anthracen-9-yl-benzo[b]thiophene (16) ${ }^{13 c}$}

From 9-bromoanthracene $(0.257 \mathrm{~g}, 1 \mathrm{mmol})$ and benzothiophene $(0.201 \mathrm{~g}, 1.5 \mathrm{mmol}), \mathbf{1 6}$ was obtained in $83 \%(0.257 \mathrm{~g})$ yield. ${ }^{1} \mathrm{H}$ NMR (400 MHz, $\left.\mathrm{CDCl}_{3}\right): \square \delta 8.49(\mathrm{~s}, 1 \mathrm{H}), 7.99(\mathrm{~d}, J=8.4 \mathrm{~Hz}$, $2 \mathrm{H}), 7.90-7.82(\mathrm{~m}, 4 \mathrm{H}), 7.45-7.30(\mathrm{~m}, 7 \mathrm{H})$.

\section{5-Benzo[b]thiophen-2-yl-pyrimidine (17)}

From 5-bromopyrimidine $(0.159 \mathrm{~g}, 1 \mathrm{mmol})$ and benzothiophene $(0.201 \mathrm{~g}, 1.5 \mathrm{mmol}), 17$ was obtained in $66 \%(0.140 \mathrm{~g})$ yield as a white solid (mp 128-130 $\left.{ }^{\circ} \mathrm{C}\right) .{ }^{1} \mathrm{H}$ NMR $\left(400 \mathrm{MHz}, \mathrm{CDCl}_{3}\right): \square \delta$ $9.09(\mathrm{~s}, 1 \mathrm{H}), 8.95(\mathrm{~s}, 2 \mathrm{H}), 7.79(\mathrm{~d}, J=7.7 \mathrm{~Hz}, 1 \mathrm{H}), 7.75(\mathrm{~d}, J=$ $7.5 \mathrm{~Hz}, 1 \mathrm{H}), 7.56(\mathrm{~s}, 1 \mathrm{H}), 7.32(\mathrm{t}, J=7.4 \mathrm{~Hz}, 1 \mathrm{H}), 7.29(\mathrm{t}, J=7.4$ $\mathrm{Hz}, 1 \mathrm{H}) .{ }^{13} \mathrm{C} \mathrm{NMR}\left(100 \mathrm{MHz}, \mathrm{CDCl}_{3}\right): \delta 157.8,153.9,140.1$, 139.9, 136.1, 128.7, 125.5, 125.1, 124.2, 122.5, 121.9. elemental analysis: calcd (\%) for $\mathrm{C}_{12} \mathrm{H}_{8} \mathrm{~N}_{2} \mathrm{~S}$ (212.27): C 67.90, H 3.80; found: C 67.99, H 3.64.

\section{4-(3-Formylbenzo[b]thiophen-2-yl)-benzonitrile $(\mathbf{1 8})^{8 \mathrm{a}}$}

From 4-bromobenzonitrile $(0.182 \mathrm{~g}, 1 \mathrm{mmol})$ and benzothiophene-3-carbaldehyde $(0.243 \mathrm{~g}, 1.5 \mathrm{mmol}), \mathbf{1 8}$ was obtained in $65 \%(0.171 \mathrm{~g})$ yield. ${ }^{1} \mathrm{H}$ NMR $\left(400 \mathrm{MHz}, \mathrm{CDCl}_{3}\right)$ : $\square$ $\delta 9.97(\mathrm{~s}, 1 \mathrm{H}), 8.71(\mathrm{~d}, J=8.1 \mathrm{~Hz}, 1 \mathrm{H}), 7.81(\mathrm{~d}, J=7.9 \mathrm{~Hz}, 1 \mathrm{H})$, $7.75(\mathrm{~d}, J=8.4 \mathrm{~Hz}, 2 \mathrm{H}), 7.64(\mathrm{~d}, J=8.4 \mathrm{~Hz}, 2 \mathrm{H}), 7.48(\mathrm{t}, J=7.3$ $\mathrm{Hz}, 1 \mathrm{H}), 7.42(\mathrm{t}, J=7.3 \mathrm{~Hz}, 1 \mathrm{H})$.

\section{2-(3-Nitrophenyl)-benzo[b]thiophene-3-carbaldehyde (19)}

From 3-bromonitrobenzene $(0.202,1 \mathrm{mmol})$ and benzothiophene3 -carbaldehyde $(0.243 \mathrm{~g}, 1.5 \mathrm{mmol}), 19$ was obtained in $78 \%$ $(0.221 \mathrm{~g})$ yield as a yellow solid (mp $\left.136-138{ }^{\circ} \mathrm{C}\right) .{ }^{1} \mathrm{H}$ NMR $(400$ $\left.\mathrm{MHz}, \mathrm{CDCl}_{3}\right): \square \delta 9.99(\mathrm{~s}, 1 \mathrm{H}), 8.72(\mathrm{~d}, J=8.1 \mathrm{~Hz}, 1 \mathrm{H}), 8.40(\mathrm{~d}$, $J=1.7 \mathrm{~Hz}, 1 \mathrm{H}), 8.32(\mathrm{~d}, J=8.3 \mathrm{~Hz}, 1 \mathrm{H}), 7.85(\mathrm{~d}, J=8.4 \mathrm{~Hz}, 1 \mathrm{H})$, $7.82(\mathrm{~d}, J=8.4 \mathrm{~Hz}, 1 \mathrm{H}), 7.4867(\mathrm{t}, J=7.3 \mathrm{~Hz}, 1 \mathrm{H}), 7.50(\mathrm{t}, J=$ $7.3 \mathrm{~Hz}, 1 \mathrm{H}), 7.45(\mathrm{t}, J=7.3 \mathrm{~Hz}, 1 \mathrm{H}) .{ }^{13} \mathrm{C} \mathrm{NMR}(100 \mathrm{MHz}$, $\left.\mathrm{CDCl}_{3}\right): \delta 185.4,156.3,148.4,138.2,136.8,136.2,133.4,131.1$, $130.0,126.7,126.5,125.4,125.1,124.6,121.8$. elemental analysis: calcd (\%) for $\mathrm{C}_{15} \mathrm{H}_{9} \mathrm{NO}_{3} \mathrm{~S}$ (283.30): C 63.59, H 3.20; found: C $63.42, \mathrm{H} 3.04$

\section{2-(3-Formylbenzo[b]thiophen-2-yl)-benzonitrile (20) ${ }^{8 \mathrm{a}}$}

From 2-bromobenzonitrile $(0.182 \mathrm{~g}, \quad 1 \mathrm{mmol})$ and benzothiophene-3-carbaldehyde $(0.243 \mathrm{~g}, 1.5 \mathrm{mmol}), 20$ was obtained in $77 \%(0.202 \mathrm{~g})$ yield. ${ }^{1} \mathrm{H}$ NMR $\left(400 \mathrm{MHz}, \mathrm{CDCl}_{3}\right)$ : $\square$ $\delta 9.83(\mathrm{~s}, 1 \mathrm{H}), 8.71(\mathrm{~d}, J=8.1 \mathrm{~Hz}, 1 \mathrm{H}), 7.81(\mathrm{~d}, J=7.8 \mathrm{~Hz}, 1 \mathrm{H})$, $7.79(\mathrm{~d}, J=7.8 \mathrm{~Hz}, 1 \mathrm{H}), 7.67(\mathrm{t}, J=7.8 \mathrm{~Hz}, 1 \mathrm{H}), 7.60-7.55(\mathrm{~m}$, $2 \mathrm{H}), 7.49(\mathrm{t}, J=7.3 \mathrm{~Hz}, 1 \mathrm{H}), 7.43(\mathrm{t}, J=7.3 \mathrm{~Hz}, 1 \mathrm{H})$.

\section{2-Isoquinolin-4-yl-benzo[b]thiophene-3-carbaldehyde (21)}

From 4-bromoisoquinoline $(0.208 \mathrm{~g}, 1 \mathrm{mmol})$ and benzothiophene-3-carbaldehyde $(0.243 \mathrm{~g}, 1.5 \mathrm{mmol}), 21$ was obtained in 65\% (0.188 g) yield as a yellow solid (mp 132-134 $\left.{ }^{\circ} \mathrm{C}\right) .{ }^{1} \mathrm{H} \mathrm{NMR}\left(400 \mathrm{MHz}, \mathrm{CDCl}_{3}\right): \square \delta 9.67(\mathrm{~s}, 1 \mathrm{H}), 9.32(\mathrm{~s}, 1 \mathrm{H})$, $8.76(\mathrm{~d}, J=8.1 \mathrm{~Hz}, 1 \mathrm{H}), 8.61(\mathrm{~s}, 1 \mathrm{H}), 8.02(\mathrm{~d}, J=7.6 \mathrm{~Hz}, 1 \mathrm{H})$, $7.83(\mathrm{~d}, J=7.6 \mathrm{~Hz}, 1 \mathrm{H}), 7.79(\mathrm{~d}, J=7.6 \mathrm{~Hz}, 1 \mathrm{H}), 7.64(\mathrm{t}, J=7.6$ $\mathrm{Hz}, 1 \mathrm{H}), 7.62(\mathrm{t}, J=7.7 \mathrm{~Hz}, 1 \mathrm{H}), 7.50(\mathrm{t}, J=7.6 \mathrm{~Hz}, 1 \mathrm{H}), 7.43(\mathrm{t}$, $J=7.6 \mathrm{~Hz}, 1 \mathrm{H}) .{ }^{13} \mathrm{C}$ NMR $\left(100 \mathrm{MHz}, \mathrm{CDCl}_{3}\right): \delta 185.8,154.4$, $154.1,144.6,139.0,136.4,135.3,133.1,132.0,128.2,128.1$, 126.6, 126.3, 125.3, 124.3, 121.7. elemental analysis: calcd (\%) for $\mathrm{C}_{18} \mathrm{H}_{11} \mathrm{NOS}$ (289.35): C 74.72, H 3.83; found: C 74.60, H 3.98 .

\section{2-Pyrimidin-5-yl-benzo[b]thiophene-3-carbaldehyde (22)}

From 5-bromopyrimidine $(0.159 \mathrm{~g}, 1 \mathrm{mmol})$ and benzothiophene3-carbaldehyde $(0.243 \mathrm{~g}, 1.5 \mathrm{mmol}), 22$ was obtained in $61 \%$ $(0.146 \mathrm{~g})$ yield as a yellow solid (mp 151-153 $\left.{ }^{\circ} \mathrm{C}\right) .{ }^{1} \mathrm{H}$ NMR (400 $\left.\mathrm{MHz}, \mathrm{CDCl}_{3}\right): \square \delta 10.00(\mathrm{~s}, 1 \mathrm{H}), 9.30(\mathrm{~s}, 1 \mathrm{H}), 8.94(\mathrm{~s}, 2 \mathrm{H}), 8.70$ $(\mathrm{d}, J=8.1 \mathrm{~Hz}, 1 \mathrm{H}), 7.83(\mathrm{~d}, J=8.1 \mathrm{~Hz}, 1 \mathrm{H}), 7.50(\mathrm{t}, J=8.1 \mathrm{~Hz}$, $1 \mathrm{H}), 7.44(\mathrm{t}, J=8.1 \mathrm{~Hz}, 1 \mathrm{H}) .{ }^{13} \mathrm{C}$ NMR $\left(100 \mathrm{MHz}, \mathrm{CDCl}_{3}\right): \delta$ $184.6,159.3,157.0,150.7,138.4,136.8,131.7,126.9,126.7$, 125.3, 121.9. elemental analysis: calcd $(\%)$ for $\mathrm{C}_{13} \mathrm{H}_{8} \mathrm{~N}_{2} \mathrm{OS}$ (240.28): C 64.98, H 3.36; found: C 65.14, H 3.47.

\section{4-(3-Methylbenzo[b]thiophen-2-yl)-benzonitrile (23)}

From 4-bromobenzonitrile $(0.182 \mathrm{~g}, 1 \mathrm{mmol})$ and 3-methylbenzothiophene $(0.222 \mathrm{~g}, 1.5 \mathrm{mmol}), 23$ was obtained in $88 \%$ $(0.219 \mathrm{~g})$ yield as a white solid $\left(\mathrm{mp} 125-127{ }^{\circ} \mathrm{C}\right) .{ }^{1} \mathrm{H}$ NMR $(400$ $\left.\mathrm{MHz}, \mathrm{CDCl}_{3}\right): \square \delta 7.76(\mathrm{~d}, J=7.5 \mathrm{~Hz}, 1 \mathrm{H}), 7.67(\mathrm{~d}, J=7.8 \mathrm{~Hz}$, $1 \mathrm{H}), 7.64(\mathrm{~d}, J=8.4 \mathrm{~Hz}, 2 \mathrm{H}), 7.55(\mathrm{~d}, J=8.4 \mathrm{~Hz}, 2 \mathrm{H}), 7.36(\mathrm{t}, J=$ $7.3 \mathrm{~Hz}, 1 \mathrm{H}), 7.30(\mathrm{t}, J=7.3 \mathrm{~Hz}, 1 \mathrm{H}), 2.40(\mathrm{~s}, 3 \mathrm{H}) .{ }^{13} \mathrm{C}$ NMR $(100$ $\left.\mathrm{MHz}, \mathrm{CDCl}_{3}\right): \delta 141.0,139.6,139.1,135.7,132.3,130.2,129.4$, 125.2, 124.6, 122.6, 122.3, 118.7, 111.3, 12.8. elemental analysis: calcd (\%) for $\mathrm{C}_{16} \mathrm{H}_{11} \mathrm{NS}$ (249.33): $\mathrm{C} 77.07, \mathrm{H} \mathrm{4.45}$; found: $\mathrm{C}$ 77.01, H 4.35 .

\section{Ethyl 4-(3-methylbenzo[b]thiophen-2-yl)-benzoate (24)}

From ethyl 4-bromobenzoate $(0.229 \mathrm{~g}, 1 \mathrm{mmol})$ and 3-methylbenzothiophene $(0.222 \mathrm{~g}, 1.5 \mathrm{mmol}), 24$ was obtained in $81 \%$ $(0.240 \mathrm{~g})$ yield as a yellow oil. ${ }^{1} \mathrm{H}$ NMR $\left(400 \mathrm{MHz}, \mathrm{CDCl}_{3}\right): \square \delta$ $8.05(\mathrm{~d}, J=8.2 \mathrm{~Hz}, 2 \mathrm{H}), 7.76(\mathrm{~d}, J=7.5 \mathrm{~Hz}, 1 \mathrm{H}), 7.67(\mathrm{~d}, J=7.8$ $\mathrm{Hz}, 1 \mathrm{H}), 7.54(\mathrm{~d}, J=8.2 \mathrm{~Hz}, 2 \mathrm{H}), 7.35(\mathrm{t}, J=7.3 \mathrm{~Hz}, 1 \mathrm{H}), 7.29(\mathrm{t}$, $J=7.3 \mathrm{~Hz}, 1 \mathrm{H}), 4.34(\mathrm{q}, J=7.1 \mathrm{~Hz}, 2 \mathrm{H}), 2.41(\mathrm{~s}, 3 \mathrm{H}), 1.34(\mathrm{t}, J=$ $7.1 \mathrm{~Hz}, 3 \mathrm{H}) .{ }^{13} \mathrm{C} \mathrm{NMR}\left(100 \mathrm{MHz}, \mathrm{CDCl}_{3}\right): \delta 166.3,141.1,139.3$, $139.1,136.8,129.8,129.6,129.5,128.6,124.7,124.3,122.4$, $122.2,61.1,14.4,12.8$. elemental analysis: calcd $(\%)$ for $\mathrm{C}_{18} \mathrm{H}_{16} \mathrm{O}_{2} \mathrm{~S}$ (296.38): C 72.94, H 5.44; found: C 72.80, H 5.32.

\section{2-(4-Chlorophenyl)-3-methylbenzo[b]thiophene (25)}

From 1-bromo-4-chlorobenzene $(0.191 \mathrm{~g}, 1 \mathrm{mmol})$ and 3-methylbenzothiophene $(0.222 \mathrm{~g}, 1.5 \mathrm{mmol}), \mathbf{2 5}$ was obtained in $88 \%$ $(0.227 \mathrm{~g})$ yield as a white solid $\left(\mathrm{mp} 87-89{ }^{\circ} \mathrm{C}\right) .{ }^{1} \mathrm{H}$ NMR $(400$ $\left.\mathrm{MHz}, \mathrm{CDCl}_{3}\right): \square 7.74(\mathrm{~d}, J=7.5 \mathrm{~Hz}, 1 \mathrm{H}), 7.64(\mathrm{~d}, J=7.8 \mathrm{~Hz}$, 
$1 \mathrm{H}), 7.39(\mathrm{~d}, J=8.2 \mathrm{~Hz}, 2 \mathrm{H}), 7.34(\mathrm{~d}, J=8.2 \mathrm{~Hz}, 2 \mathrm{H}), 7.31(\mathrm{t}, J=$ $7.3 \mathrm{~Hz}, 1 \mathrm{H}), 7.27(\mathrm{t}, J=7.3 \mathrm{~Hz}, 1 \mathrm{H}), 2.36(\mathrm{~s}, 3 \mathrm{H}) .{ }^{13} \mathrm{C} \mathrm{NMR}(100$ $\left.\mathrm{MHz}, \mathrm{CDCl}_{3}\right): \delta 141.1,138.9,136.7,133.9,133.2,130.9,128.8$, 127.9, 124.5, 124.3, 122.2, 122.1, 12.6. elemental analysis: calcd (\%) for $\mathrm{C}_{15} \mathrm{H}_{11} \mathrm{ClS}$ (258.77): C 69.62, H 4.28; found: C 69.79, $\mathrm{H}$ 4.09.

\section{3-Methyl-2-m-tolyl-benzo[b]thiophene (26)}

From 3-bromotoluene $(0.171 \mathrm{~g}, 1 \mathrm{mmol})$ and 3-methylbenzothiophene $(0.222 \mathrm{~g}, 1.5 \mathrm{mmol}), 26$ was obtained in $66 \%$ $(0.157 \mathrm{~g})$ yield as a colourless oil. ${ }^{1} \mathrm{H}$ NMR $\left(400 \mathrm{MHz}, \mathrm{CDCl}_{3}\right)$ : $\square$ $\delta 7.72(\mathrm{~d}, J=7.8 \mathrm{~Hz}, 1 \mathrm{H}), 7.61(\mathrm{~d}, J=7.9 \mathrm{~Hz}, 1 \mathrm{H}), 7.35-7.18(\mathrm{~m}$, $5 \mathrm{H}), 7.10-7.05(\mathrm{~m}, 1 \mathrm{H}), 2.36(\mathrm{~s}, 3 \mathrm{H}), 2.32(\mathrm{~s}, 3 \mathrm{H}) .{ }^{13} \mathrm{C}$ NMR $(100$ $\left.\mathrm{MHz}, \mathrm{CDCl}_{3}\right): \delta 141.3,138.9,138.3,138.2,134.7,130.4,128.6$, $128.5,127.3,126.9,124.3,124.1,122.2,122.1,21.5,12.7$ elemental analysis: calcd (\%) for $\mathrm{C}_{16} \mathrm{H}_{14} \mathrm{~S}$ (238.35): $\mathrm{C} 80.63, \mathrm{H}$ 5.92; found: C 80.74, H 5.99.

\section{2-(3-Methylbenzo[b]thiophen-2-yl)-benzonitrile (27)}

From 2-bromobenzonitrile $(0.182 \mathrm{~g}, 1 \mathrm{mmol})$ and 3-methylbenzothiophene $(0.222 \mathrm{~g}, 1.5 \mathrm{mmol}), 27$ was obtained in $79 \%$ $(0.197 \mathrm{~g})$ yield as a white solid (mp 122-124 $\left.{ }^{\circ} \mathrm{C}\right) .{ }^{1} \mathrm{H}$ NMR (400 $\left.\mathrm{MHz}_{\mathrm{CDCl}}\right): \square \delta 7.75(\mathrm{~d}, J=7.5 \mathrm{~Hz}, 1 \mathrm{H}), 7.70(\mathrm{~d}, J=7.8 \mathrm{~Hz}$, $1 \mathrm{H}), 7.68(\mathrm{~d}, J=7.8 \mathrm{~Hz}, 1 \mathrm{H}), 7.56(\mathrm{t}, J=7.8 \mathrm{~Hz}, 1 \mathrm{H}), 7.48(\mathrm{~d}, J=$ $7.2 \mathrm{~Hz}, 1 \mathrm{H}), 7.41(\mathrm{t}, J=7.3 \mathrm{~Hz}, 1 \mathrm{H}), 7.36(\mathrm{t}, J=7.3 \mathrm{~Hz}, 1 \mathrm{H}), 7.31$ $(\mathrm{t}, J=7.3 \mathrm{~Hz}, 1 \mathrm{H}), 2.30(\mathrm{~s}, 3 \mathrm{H}) .{ }^{13} \mathrm{C} \mathrm{NMR}\left(100 \mathrm{MHz}, \mathrm{CDCl}_{3}\right): \delta$ $140.3,139.6,138.4,133.3,133.1,132.5,132.0,131.2,128.6$, 125.1, 124.5, 122.7, 122.2, 117.9, 113.9, 13.0. elemental analysis:

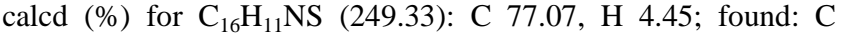
77.40, H 4.57 .

\section{3-(3-Methylbenzo[b]thiophen-2-yl)-pyridine (28)}

From 3-bromopyridine $(0.158 \mathrm{~g}, 1 \mathrm{mmol})$ and 3-methylbenzothiophene $(0.222 \mathrm{~g}, 1.5 \mathrm{mmol}), 28$ was obtained in $70 \%$ $(0.157 \mathrm{~g})$ yield as a white solid (mp 210-212 $\left.{ }^{\circ} \mathrm{C}\right) .{ }^{1} \mathrm{H}$ NMR $(400$ $\left.\mathrm{MHz}, \mathrm{CDCl}_{3}\right): \square \delta 8.73(\mathrm{~d}, J=1.2 \mathrm{~Hz}, 1 \mathrm{H}), 8.53(\mathrm{~d}, J=3.6 \mathrm{~Hz}$, $1 \mathrm{H}), 7.80-7.70(\mathrm{~m}, 2 \mathrm{H}), 7.66(\mathrm{~d}, J=8.0 \mathrm{~Hz}, 1 \mathrm{H}), 7.40-7.23(\mathrm{~m}$, $3 \mathrm{H}), 2.39(\mathrm{~s}, 3 \mathrm{H}) .{ }^{13} \mathrm{C}$ NMR $\left(100 \mathrm{MHz}, \mathrm{CDCl}_{3}\right): \delta 150.2,148.8$, $140.9,139.1,136.8,133.9,130.9,129.0,124.8,124.4,123.4$, 122.4, 122.2, 12.6. elemental analysis: calcd $(\%)$ for $\mathrm{C}_{14} \mathrm{H}_{11} \mathrm{NS}$ (225.31): C 74.63, H 4.92; found: C 74.57, H 4.69.

\section{4-(3-Methylbenzo[b]thiophen-2-yl)-isoquinoline (29)}

From 4-bromoisoquinoline $(0.208 \mathrm{~g}, 1 \mathrm{mmol})$ and 3-methylbenzothiophene $(0.222 \mathrm{~g}, 1.5 \mathrm{mmol}), 29$ was obtained in $61 \%$ $(0.168 \mathrm{~g})$ yield as a yellow oil. ${ }^{1} \mathrm{H}$ NMR $\left(400 \mathrm{MHz}, \mathrm{CDCl}_{3}\right): \square \delta$ $9.24(\mathrm{~s}, 1 \mathrm{H}), 8.53(\mathrm{~s}, 1 \mathrm{H}), 7.99(\mathrm{~d}, J=8.0 \mathrm{~Hz}, 1 \mathrm{H}), 7.81(\mathrm{~d}, J=$ 7.7 Hz, 1H), 7.75-7.70 (m, 2H), 7.65-7.55 (m, 2H), 7.40 (t, $J=7.8$ $\mathrm{Hz}, 1 \mathrm{H}), 7.33(\mathrm{t}, J=7.8 \mathrm{~Hz}, 1 \mathrm{H}), 2.16(\mathrm{~s}, 3 \mathrm{H}),{ }^{13} \mathrm{C}$ NMR $(100$ $\left.\mathrm{MHz}, \mathrm{CDCl}_{3}\right): \delta 153.0,144.8,140.4,139.9,135.2,131.9,131.3$, $131.0,128.3,128.0,127.5,125.9,125.0,124.7,124.4,122.3$, 122.2, 12.7. elemental analysis: calcd (\%) for $\mathrm{C}_{18} \mathrm{H}_{13} \mathrm{NS}(275.37)$ : C 78.51, H 4.76; found: C 78.51, H 4.87.

\section{4-(5-Chloro-3-methylbenzo[b]thiophen-2-yl)-benzonitrile (30)} From 4-bromobenzonitrile $(0.182 \mathrm{~g}, 1 \mathrm{mmol})$ and 5-chloro-3methylbenzothiophene $(0.274 \mathrm{~g}, 1.5 \mathrm{mmol}), 30$ was obtained in $78 \%(0.221 \mathrm{~g})$ yield as a white solid (mp 164-166 $\left.{ }^{\circ} \mathrm{C}\right) .{ }^{1} \mathrm{H}$ NMR $\left(400 \mathrm{MHz}, \mathrm{CDCl}_{3}\right): \square \delta 7.70-7.63(\mathrm{~m}, 4 \mathrm{H}), 7.56(\mathrm{~d}, J=8.3 \mathrm{~Hz}$, $2 \mathrm{H}), 7.27(\mathrm{~d}, J=8.5 \mathrm{~Hz}, 1 \mathrm{H}), 2.37(\mathrm{~s}, 3 \mathrm{H}) .{ }^{13} \mathrm{C} \mathrm{NMR}(100 \mathrm{MHz}$, $\left.\mathrm{CDCl}_{3}\right): \delta 142.1,139.0,137.7,137.2,132.4,130.9,130.2,128.7$, 125.5, 123.3, 122.2, 118.6, 111.7, 12.8. elemental analysis: calcd (\%) for $\mathrm{C}_{16} \mathrm{H}_{10} \mathrm{ClNS}$ (283.78): C 67.72, H 3.55; found: C 67.81, H 3.41 .
1-[4-(5-Chloro-3-methylbenzo[b]thiophen-2-yl)-phenyl]propan-1-one (31)

From 4-bromopropiophenone $(0.213 \mathrm{~g}, 1 \mathrm{mmol})$ and 5-chloro-3methylbenzothiophene $(0.274 \mathrm{~g}, 1.5 \mathrm{mmol}), 31$ was obtained in $83 \%(0.261 \mathrm{~g})$ yield as a white solid (mp 136-138 $\left.{ }^{\circ} \mathrm{C}\right) .{ }^{1} \mathrm{H}$ NMR (400 MHz, $\left.\mathrm{CDCl}_{3}\right): \square \delta 7.94(\mathrm{~d}, J=8.3 \mathrm{~Hz}, 2 \mathrm{H}), 7.62-7.58(\mathrm{~m}$, $2 \mathrm{H}), 7.51(\mathrm{~d}, J=8.3 \mathrm{~Hz}, 2 \mathrm{H}), 7.27(\mathrm{~d}, J=8.5 \mathrm{~Hz}, 1 \mathrm{H}), 2.91(\mathrm{q}, J$ $=7.5 \mathrm{~Hz}, 2 \mathrm{H}), 2.34(\mathrm{~s}, 3 \mathrm{H}), 1.16(\mathrm{t}, J=7.5 \mathrm{~Hz}, 3 \mathrm{H}) .{ }^{13} \mathrm{C} \mathrm{NMR}$ $\left(100 \mathrm{MHz}, \mathrm{CDCl}_{3}\right): \delta 200.1,142.4,138.8,137.1,136.1,130.7$, $129.7,128.3,128.1,125.1,123.2,122.1,31.9,12.8,8.3$. elemental analysis: calcd (\%) for $\mathrm{C}_{18} \mathrm{H}_{15} \mathrm{ClOS}$ (314.83): C 68.67, H 4.80; found: C 68.49, H 4.89 .

\section{5-Chloro-2-(4-chlorophenyl)-3-methylbenzo[b]thiophene (32)}

From 1-bromo-4-chlorobenzene $(0.191 \mathrm{~g}, 1 \mathrm{mmol})$ and 5-chloro3-methylbenzothiophene $(0.274 \mathrm{~g}, 1.5 \mathrm{mmol}), 32$ was obtained in $85 \%(0.249 \mathrm{~g})$ yield as a white solid (mp 137-138 $\left.{ }^{\circ} \mathrm{C}\right) .{ }^{1} \mathrm{H}$ NMR $\left(400 \mathrm{MHz}, \mathrm{CDCl}_{3}\right): \square \delta 7.60(\mathrm{~d}, J=8.2 \mathrm{~Hz}, 1 \mathrm{H}), 7.58(\mathrm{~s}, 1 \mathrm{H})$, 7.40-7.30 (m, 4H), $7.20(\mathrm{~d}, J=8.3 \mathrm{~Hz}, 1 \mathrm{H}), 2.30(\mathrm{~s}, 3 \mathrm{H}) .{ }^{13} \mathrm{C}$ NMR $\left(100 \mathrm{MHz}, \mathrm{CDCl}_{3}\right): \delta 142.4,138.7,136.9,134.2,132.7$, 130.9, 130.7, 128.9, 127.4, 124.9, 123.2, 121.9, 12.6. elemental analysis: calcd (\%) for $\mathrm{C}_{15} \mathrm{H}_{10} \mathrm{Cl}_{2} \mathrm{~S}$ (293.21): $\mathrm{C}$ 61.44, $\mathrm{H}$ 3.44; found: C $61.59, \mathrm{H} 3.31$.

\section{2-(4-tert-Butylphenyl)-3-methyl-benzo[b]thiophene (33)}

From 1-tert-butylbromobenzene $(0.213 \mathrm{~g}, 1 \mathrm{mmol})$ and 5-chloro3-methylbenzothiophene $(0.274 \mathrm{~g}, 1.5 \mathrm{mmol}), 33$ was obtained in $58 \%(0.182 \mathrm{~g})$ yield as a white solid (mp 134-136 $\left.{ }^{\circ} \mathrm{C}\right) .{ }^{1} \mathrm{H}$ NMR $\left(400 \mathrm{MHz}, \mathrm{CDCl}_{3}\right): \square \delta 7.64(\mathrm{~d}, J=8.2 \mathrm{~Hz}, 1 \mathrm{H}), 7.60(\mathrm{~s}, 1 \mathrm{H})$, 7.45-7.35 (m, 4H), $7.22(\mathrm{~d}, J=8.3 \mathrm{~Hz}, 1 \mathrm{H}), 2.36(\mathrm{~s}, 3 \mathrm{H}), 1.30(\mathrm{~s}$, 9H). $\left.{ }^{13} \mathrm{C} \mathrm{NMR} \mathrm{(100} \mathrm{MHz,} \mathrm{CDCl}_{3}\right): \delta 151.2,142.6,140.3,136.9$, 131.3, 130.4, 129.3, 126.6, 125.6, 124.4, 123.1, 121.7, 34.8, 31.3, 12.6. elemental analysis: calcd (\%) for $\mathrm{C}_{19} \mathrm{H}_{20} \mathrm{ClS}$ (314.87): $\mathrm{C}$ 72.47, H 6.08; found: C 72.27, H 6.29.

\section{5-Chloro-3-methyl-2-(3-nitrophenyl)-benzo[b]thiophene (34)} From 3-bromonitrobenzene $(0.202,1 \mathrm{mmol})$ and 5-chloro-3methylbenzothiophene $(0.274 \mathrm{~g}, 1.5 \mathrm{mmol}), 34$ was obtained in $83 \%(0.251 \mathrm{~g})$ yield as a yellow solid $\left(\mathrm{mp} 176-177^{\circ} \mathrm{C}\right) .{ }^{1} \mathrm{H}$ NMR $\left(400 \mathrm{MHz}, \mathrm{CDCl}_{3}\right): \square \delta 8.30(\mathrm{~s}, 1 \mathrm{H}), 8.16(\mathrm{~d}, J=8.3 \mathrm{~Hz}, 1 \mathrm{H})$, $7.75(\mathrm{~d}, J=7.7 \mathrm{~Hz}, 1 \mathrm{H}), 7.68-7.60(\mathrm{~m}, 2 \mathrm{H}), 7.57(\mathrm{t}, J=8.0 \mathrm{~Hz}$, $1 \mathrm{H}), 7.26(\mathrm{~d}, J=8.5 \mathrm{~Hz}, 1 \mathrm{H}), 2.37(\mathrm{~s}, 3 \mathrm{H}) .{ }^{13} \mathrm{C} \mathrm{NMR}(100 \mathrm{MHz}$, $\left.\mathrm{CDCl}_{3}\right): \delta 148.4,142.1,137.0,136.0,135.5,131.0,129.7,128.7$, $125.5,124.3,123.3,122.8,122.2,12.6$. elemental analysis: calcd (\%) for $\mathrm{C}_{15} \mathrm{H}_{10} \mathrm{ClNO}_{2} \mathrm{~S}$ (303.76): C 59.31, H 3.32; found: C 59.50, H 3.48 .

\section{5-Chloro-3-methyl-2-m-tolyl-benzo[b]thiophene (35)}

From 3-bromotoluene $(0.171 \mathrm{~g}, 1 \mathrm{mmol})$ and 5-chloro-3methylbenzothiophene $(0.274 \mathrm{~g}, 1.5 \mathrm{mmol}), 35$ was obtained in $80 \%(0.218 \mathrm{~g})$ yield as a colourless oil. ${ }^{1} \mathrm{H}$ NMR $(400 \mathrm{MHz}$, $\left.\mathrm{CDCl}_{3}\right): \square \delta .58(\mathrm{~d}, J=8.3 \mathrm{~Hz}, 1 \mathrm{H}), 7.56(\mathrm{~s}, 1 \mathrm{H}), 7.30-7.05(\mathrm{~m}$, $5 \mathrm{H}), 2.31(\mathrm{~s}, 3 \mathrm{H}), 2.30(\mathrm{~s}, 3 \mathrm{H}) .{ }^{13} \mathrm{C} \mathrm{NMR}\left(100 \mathrm{MHz}, \mathrm{CDCl}_{3}\right): \delta$ $142.6,140.4,138.4,137.0,134.2,130.5,130.3,128.9$, 128.6, $126.8,126.7,124.6,123.1,121.8,21.5,12.7$. elemental analysis: calcd (\%) for $\mathrm{C}_{16} \mathrm{H}_{13} \mathrm{ClS}$ (272.79): $\mathrm{C} 70.45, \mathrm{H} 4.80$; found: $\mathrm{C}$ 70.32, H 4.68 .

5-Chloro-3-methyl-2-naphthalen-2-yl-benzo[b]thiophene (36) From 2-bromonaphthalene $(0.207 \mathrm{~g}, 1 \mathrm{mmol})$ and 5-chloro-3methylbenzothiophene $(0.274 \mathrm{~g}, 1.5 \mathrm{mmol}), 36$ was obtained in $88 \%(0.271 \mathrm{~g})$ yield as a white solid (mp 128-129 $\left.{ }^{\circ} \mathrm{C}\right) .{ }^{1} \mathrm{H}$ NMR $\left(400 \mathrm{MHz}, \mathrm{CDCl}_{3}\right): \square \delta 7.85(\mathrm{~s}, 1 \mathrm{H}), 7.80-7.70(\mathrm{~m}, 3 \mathrm{H}), 7.65-7.55$ $(\mathrm{m}, 2 \mathrm{H}), 7.51(\mathrm{~d}, J=8.3 \mathrm{~Hz}, 1 \mathrm{H}), 7.43-7.38(\mathrm{~m}, 2 \mathrm{H}), 7.19(\mathrm{~d}, J=$ $8.4 \mathrm{~Hz}, 1 \mathrm{H}), 2.34(\mathrm{~s}, 3 \mathrm{H}) .{ }^{13} \mathrm{C}$ NMR $\left(100 \mathrm{MHz}, \mathrm{CDCl}_{3}\right): \delta 142.6$, 
$140.2,137.2,133.3,132.8,131.7,130.6,128.9,128.3,128.2$, $127.8,127.3,127.2,126.7,126.6,124.7,123.2,121.9,12.8$. elemental analysis: calcd (\%) for $\mathrm{C}_{19} \mathrm{H}_{13} \mathrm{ClS}$ (308.83): $\mathrm{C} 73.89, \mathrm{H}$ 4.24; found: C 74.08, H 4.01.

2-(5-Chloro-3-methyl-benzo[b]thiophen-2-yl)-benzonitrile (37) From 2-bromobenzonitrile $(0.182 \mathrm{~g}, 1 \mathrm{mmol})$ and 5-chloro-3methylbenzothiophene $(0.274 \mathrm{~g}, 1.5 \mathrm{mmol}), 37$ was obtained in $82 \%(0.232 \mathrm{~g})$ yield as a white solid $\left(\mathrm{mp} 161-163{ }^{\circ} \mathrm{C}\right) .{ }^{1} \mathrm{H}$ NMR $\left(400 \mathrm{MHz}, \mathrm{CDCl}_{3}\right): \square \delta 7.71(\mathrm{~d}, J=8.3 \mathrm{~Hz}, 1 \mathrm{H}), 7.68-7.60(\mathrm{~m}$, $2 \mathrm{H}), 7.58(\mathrm{t}, J=7.7 \mathrm{~Hz}, 1 \mathrm{H}), 7.50-7.40(\mathrm{~m}, 2 \mathrm{H}), 7.25(\mathrm{~d}, J=8.6$ $\mathrm{Hz}, 1 \mathrm{H}), 2.25(\mathrm{~s}, 3 \mathrm{H}),{ }^{13} \mathrm{C}$ NMR $\left(100 \mathrm{MHz}, \mathrm{CDCl}_{3}\right): \delta 141.5$, $137.8,137.6,135.1,133.4,132.6,131.9,130.8,130.6,128.9$, $125.5,123.2,122.4,117.8,113.8,12.9$. elemental analysis: calcd (\%) for $\mathrm{C}_{16} \mathrm{H}_{10} \mathrm{ClNS}$ (283.78): $\mathrm{C} 67.72, \mathrm{H} 3.55$; found: $\mathrm{C} 67.57, \mathrm{H}$ 3.59 .

\section{3-(5-Chloro-3-methylbenzo[b]thiophen-2-yl)-pyridine (38)}

From 3-bromopyridine $(0.158 \mathrm{~g}, 1 \mathrm{mmol})$ and 5-chloro-3methylbenzothiophene $(0.274 \mathrm{~g}, 1.5 \mathrm{mmol}), 38$ was obtained in $76 \%(0.197 \mathrm{~g})$ yield as a white solid (mp 116-118 $\left.{ }^{\circ} \mathrm{C}\right) .{ }^{1} \mathrm{H}$ NMR $\left(400 \mathrm{MHz}, \mathrm{CDCl}_{3}\right): \square \delta 8.58(\mathrm{~d}, J=1.1 \mathrm{~Hz}, 1 \mathrm{H}), 8.52(\mathrm{~d}, J=3.8$ $\mathrm{Hz}, 1 \mathrm{H}), 7.69(\mathrm{~d}, J=7.9 \mathrm{~Hz}, 1 \mathrm{H}), 7.60-7.55(\mathrm{~m}, 2 \mathrm{H}), 7.27(\mathrm{dd}, J=$ $7.7,4.9 \mathrm{~Hz}, 1 \mathrm{H}), 7.20(\mathrm{dd}, J=8.5,1.8 \mathrm{~Hz}, 1 \mathrm{H}), 2.30(\mathrm{~s}, 3 \mathrm{H}) .{ }^{13} \mathrm{C}$ NMR $\left(100 \mathrm{MHz}, \mathrm{CDCl}_{3}\right): \delta 150.1,149.2,142.1,137.1,136.7$, $136.0,130.8,130.4,128.4,125.1,123.4,123.2,122.1,12.5$. elemental analysis: calcd (\%) for $\mathrm{C}_{14} \mathrm{H}_{10}$ CINS (259.75): C 64.73, H 3.88; found: C 64.50, H 3.99.

\section{2-(3-Bromobenzothiophen-2-yl)-benzonitrile (39)}

From 2-bromobenzonitrile $(0.182 \mathrm{~g}, 1 \mathrm{mmol})$, 3-bromobenzothiophene $(0.319 \mathrm{~g}, 1.5 \mathrm{mmol}), \mathrm{KOAc}(0.196 \mathrm{~g}, 2 \mathrm{mmol})$ at $150{ }^{\circ} \mathrm{C}$ during $16 \mathrm{~h}$ in DMAc $(4 \mathrm{~mL})$ in the presence of $\mathrm{Pd}(\mathrm{OAc})_{2}$ $(1.12 \mathrm{mg}, 0.005 \mathrm{mmol}), 40$ was obtained in $62 \%(0.195 \mathrm{~g})$ yield as a brown solid (mp 174-176 $\left.{ }^{\circ} \mathrm{C}\right) .{ }^{1} \mathrm{H}$ NMR (400 MHz, $\left.\mathrm{CDCl}_{3}\right)$ : $\square \delta$ $7.83(\mathrm{~d}, J=8.0 \mathrm{~Hz}, 1 \mathrm{H}), 7.77(\mathrm{~d}, J=8.0 \mathrm{~Hz}, 1 \mathrm{H}), 7.75(\mathrm{~d}, J=8.0$ $\mathrm{Hz}, 1 \mathrm{H}), 7.63(\mathrm{td}, J=7.7,1.1 \mathrm{~Hz}, 1 \mathrm{H}), 7.55(\mathrm{~d}, J=7.2 \mathrm{~Hz}, 1 \mathrm{H})$, $7.49(\mathrm{td}, J=7.7,1.1 \mathrm{~Hz}, 1 \mathrm{H}), 7.45(\mathrm{t}, J=7.7 \mathrm{~Hz}, 1 \mathrm{H}), 7.39(\mathrm{t}, J=$ $7.7 \mathrm{~Hz}, 1 \mathrm{H}) .{ }^{13} \mathrm{C} \mathrm{NMR}\left(100 \mathrm{MHz}, \mathrm{CDCl}_{3}\right): \delta 138.6,138.1,136.7$, $133.8,133.5,132.6,132.0,129.4,126.2,125.6,124.1,122.3$, 117.5, 114.0, 109.1. elemental analysis: calcd (\%) for $\mathrm{C}_{15} \mathrm{H}_{8} \mathrm{BrNS}$ (314.20): C 57.34, H 2.57; found: C 57.50, H 2.86.

\section{4-[2-(4-Chlorophenyl)-benzo[b]thiophen-3-yl]-benzonitrile (40)}

From 2-(4-chlorophenyl)-benzothiophene 7 (0.244 g, $1 \mathrm{mmol})$, 4bromobenzonitrile $(0.364 \mathrm{~g}, 2 \mathrm{mmol}), \mathrm{KOAc}(0.392 \mathrm{~g}, 4 \mathrm{mmol})$ at $150{ }^{\circ} \mathrm{C}$ during $20 \mathrm{~h}$ in DMAc $(4 \mathrm{~mL})$ in the presence of $\mathrm{PdCl}\left(\mathrm{C}_{3} \mathrm{H}_{5}\right)(\mathrm{dppb})(3.1 \mathrm{mg}, 0.005 \mathrm{mmol}) \mathbf{4 0}$ was obtained in $47 \%$ $(0.162 \mathrm{~g})$ yield as a white solid $\left(\mathrm{mp} 178-180{ }^{\circ} \mathrm{C}\right) .{ }^{1} \mathrm{H}$ NMR $(400$ $\left.\left.\mathrm{MHz}_{\mathrm{CDCl}}\right)\right) \square \delta 7.82(\mathrm{~d}, J=7.0 \mathrm{~Hz}, 1 \mathrm{H}), 7.64(\mathrm{~d}, J=8.3 \mathrm{~Hz}$, $2 \mathrm{H}), 7.46(\mathrm{~d}, J=7.0 \mathrm{~Hz}, 1 \mathrm{H}), 7.37(\mathrm{~d}, J=8.3 \mathrm{~Hz}, 2 \mathrm{H}), 7.35-7.25$ $(\mathrm{m}, 2 \mathrm{H}), 7.19(\mathrm{~d}, J=8.5 \mathrm{~Hz}, 2 \mathrm{H}), 7.12(\mathrm{~d}, J=8.5 \mathrm{~Hz}, 2 \mathrm{H}) .{ }^{13} \mathrm{C}$ NMR (100 MHz, $\left.\mathrm{CDCl}_{3}\right): \delta 140.3,139.8,139.7,139.0,134.5$, $132.6,132.0,131.6,131.2,130.9,128.9,125.2,125.0,122.8$, 122.4, 118.7, 111.4. elemental analysis: calcd (\%) for $\mathrm{C}_{21} \mathrm{H}_{12} \mathrm{ClNS}$ (345.85): C 72.93, H 3.50; found: C 72.80, H 3.66.

\section{Supporting information}

Copies of ${ }^{1} \mathrm{H}$ and ${ }^{13} \mathrm{C}$ NMR spectra of new compounds.

\section{Acknowledgments}

We are grateful to the "Chinese Scholarship Council" for a grant to Z. L. We thank the CNRS, "Région Bretagne" and "Rennes Métropole" for providing financial support.

\section{References}

1. Ohta, A.; Akita, Y.; Ohkuwa, T.; Chiba, M.; Fukunaga, R.; Miyafuji, A.; Nakata, T.; Tani, N.; Aoyagi, Y. Heterocycles 1990, 31, 1951-1958;

2. For reviews on palladium-catalyzed $\mathrm{C}-\mathrm{H}$ bond functionalization: (a) Satoh, T.; Miura, M. Chem. Lett. 2007, 36, 200205; (b) Campeau, L.-C.; Stuart, D. R.; Fagnou, K. Aldrichim. Acta 2007, 40, 35-41; (c) Seregin, I. V.; Gevorgyan, V. Chem. Soc. Rev. 2007, 36, 1173-1193; (d) Li, B.-J.; Yang, S.-D.; Shi, Z.J. Synlett 2008, 949-957; (e) Bellina, F.; Rossi, R. Tetrahedron 2009, 65, 10269-10310; (f) Ackermann, L.; Vincente, R.; Kapdi, A. R. Angew. Chem. Int. Ed. 2009, 48, 9792-9826; (g) Chen, X.; Engle, K. M.; Wang, D.-H.; Yu, J.-Q. Angew. Chem. Int. Ed. 2009, 48, 5094-5115; (h) Roger, J.; Gottumukkala, A. L.; Doucet, H. ChemCatChem 2010, 2, 20-40; (i) Fischmeister, C.; Doucet H. Green. Chem. 2011, 13, 741-753; (j) Wencel-Delord J.; Glorius, F. Nature Chem. 2013, 5, 369-375.

3. (a) Masui, K.; Ikegami, H.; Mori, A. J. Am. Chem. Soc. 2004, 126, 5074-5075; (b) Parisien, M.; Valette, D.; Fagnou, K. J. Org. Chem. 2005, 70, 7578-7584; (c) Bellina, F.; Cauteruccio, S.; Rossi, R. Eur. J. Org. Chem., 2006, 1379-1382; (d) Turner, G. L.; Morris, J. A.; Greaney, M. F. Angew. Chem., Int. Ed. 2007, 46, 7996-8000; (e) Campeau, L.-C.; Bertrand-Laperle, M.; Leclerc, J.P.; Villemure, E.; Gorelsky, S.; Fagnou, K. J. Am. Chem. Soc. 2008, 130, 3276-3277; (f) Besselievre, F.; Mahuteau-Betzer, F.; Grierson, D. S.; Piguel, S. J. Org. Chem. 2008, 73, 3278-3280; (g) Schipper, D. J.; Fagnou, K. Chem. Mater. 2011, 23, 1594-1600; (h) Nadres, E. T.; Lazareva, A.; Daugulis, O. J. Org.Chem. 2011, 76, 471-483; (i) Dröge, T.; Notzon, A.; Fröhlich, R.; Glorius, F. Chem. Eur. J. 2011, 17, 11974-11977; (j) Pierre, C.; Baudoin, O. Org. Lett. 2011, 13, 1816-1819; (k) Théveau, L.; Verrier, C.; Lassalas, P.; Martin, T.; Dupas, G.; Querolle, O.; Van Hijfte, L.; Marsais, F.; Hoarau, C. Chem. Eur. J. 2011, 17, 14450-14463.

4. For selected recent contributions on direct arylations of heteroaromatics from our laboratory: (a) Derridj, F.; Roger, J.; Djebbar, S.; Doucet, H. Org. Lett. 2010, 12, 4320-4323; (b) Chen, L.; Roger, J.; Bruneau, C.; Dixneuf, P. H.; Doucet, H. Chem. Commun. 2011, 47, 1872-1874; (c) Fu, H. Y.; Doucet H. Eur. J. Org. Chem. 2011, 7163-7173; (d) Beydoun, K.; Zaarour, M.; Williams, J. A. G.; Doucet, H.; Guerchais, V. Chem. Commun. 2012, 48, 1260-1262; (e) Derridj, F.; Roger, J.; Djebbar, S.; Doucet, H. Adv. Synth. Catal. 2012, 354, 747-750; (f) Bensaid, S.; Doucet, H. ChemSusChem 2012, 5, 1559-1567.

5. For selected examples of palladium-catalyzed direct arylation of thiophenes: (a) Okazawa, T.; Satoh, T.; Miura, M.; Nomura, M. J. Am. Chem. Soc. 2002, 124, 5286-5287; (b) Kobayashi, K.; Sugie, A.; Takahashi, M.; Masui, K.; Mori, A. Org. Lett. 2005, 7, 5083-5085; (c) Nakano, M.; Tsurugi, H.; Satoh, T.; Miura, M. Org. Lett. 2008, 10, 1851-1854; (d) Si Larbi, K.; Djebbar, S.; Doucet, H. Eur. J. Inorg. Chem. 2011, 3493-3502; (e) Beydoun, K.; Doucet, H. Eur J. Org. Chem. 2012, 6745-6751.

6. (a) Li, J. J.; Gribble, G. W. Palladium in Heterocyclic Chemistry, Pergamon: Amsterdam, 2000; (b) Negishi, E. Ed. Handbook of Organopalladium Chemistry for Organic Synthesis; Wiley-Interscience: New York, 2002; Part III, p 213. 
7. For examples of intermolecular direct arylation of benzothiophenes using palladium associated to phosphine ligands as catalyst: (a) Pivsa-Art, S.; Satoh, T.; Kawamura, Y.; Miura, M.; Nomura, M. Bull. Chem. Soc. Jpn. 1998, 71, 467-473; (b) Nakano, M.; Satoh, T.; Miura, M. J. Org. Chem. 2006, 71, 83098311; (c) Fournier Dit Chabert, J.; Chatelain, G.; Pellet-Rostaing, S.; Bouchu, D.; Lemaire, M. Tetrahedron Lett. 2006, 47, 10151018; (d) Chiong, H. A.; Daugulis, O. Org. Lett. 2007, 9, 14491451; (e) David, E.; Pellet-Rostaing, S.; Lemaire, M. Tetrahedron 2007, 63, 8999-9006; (f) Watanabe, H.; Kumagai, J.; Tsurugi, H.; Satoh, T.; Miura, M. Chem. Lett. 2007, 36, 1336-1337; (g) David, E.; Lejeune, J.; Pellet-Rostaing, S.; Schulz, J.; Lemaire, M.; Chauvin, J.; Deronzier, A. Tetrahedron Lett. 2008, 49, 18601864; (h) Liegault, B.; Lapointe, D.; Caron, L.; Vlassova, A.; Fagnou, K. J. Org. Chem. 2009, 74, 1826-1834; (i) Rene, O.; Fagnou, K. Adv. Synth. Catal. 2010, 352, 2116-2120; (j) Tamba, S.; Okubo, Y.; Tanaka, S.; Monguchi, D.; Mori, A. J. Org. Chem. 2010, 75, 6998-7001; (k) Lapointe, D.; Markiewicz, T.; Whipp, C. J.; Toderian, A.; Fagnou, K. J. Org. Chem. 2011, 76, 749-759; (1) Baghbanzadeh, M.; Pilger, C.; Kappe, C. O. J. Org. Chem. 2011, 76, 8138-8142; (m) Takeda, D.; Yamashita, M.; Hirano, K.; Satoh, T.; Miura, M. Chem. Lett. 2011, 40, 1015-1017.

8. For examples of intermolecular direct arylation of benzothiophenes using $5 \mathrm{~mol}-\% \quad \mathrm{Pd}(\mathrm{OAc})_{2}$ associated to a stoechiometric amount of quaternary ammonium salts or crown ethers as stabilizing agents: (a) Fournier Dit Chabert, J.; Joucla, L.; David, E.; Lemaire, M. Tetrahedron 2004, 60, 3221-3230; (b) David, E.; Perrin, J.; Pellet-Rostaing, S.; Fournier dit Chabert, J.; Lemaire, M. J. Org. Chem. 2005, 70, 3569-3573; (c) David, E.; Rangheard, C.; Pellet-Rostaing, S.; Lemaire, M. Synlett 2006, 2016-2020; (d) Fournier dit Chabert, J.; Marquez, B.; Neville, L.; Joucla, L.; Broussous, S.; Bouhours, P.; David, E.; PelletRostaing, S.; Marquet, B.; Moreau, N.; Lemaire, M. Bioorg. Med. Chem. 2007, 15, 4482-4497.

9. (a) de Vries, A. H. M.; Mulders, J. M. C. A.; Mommers, J. H. M.; Henderickx, H. J. W.; de Vries, J. G. Org. Lett. 2003, 5, 3285-3288; (b) Reetz, M. T.; de Vries, J. G. Chem. Commun. 2004, 15591563; (c) Alimardanov, A.; Schmieder-van de Vondervoort, L.; de Vries, A. H. M.; de Vries, J. G. Adv. Synth. Catal. 2004, 346, 1812-1817; (d) de Vries, J. G. Dalton Trans. 2006, 421-429.

10. For direct arylations of heteroaromatics with aryl halides using ligand-less palladium catalysts: (a) Parisien, M.; Valette, D.; Fagnou, K. J. Org. Chem. 2005, 70, 7578-7584; (b) Wang, X.; Gribkov, D. V.; Sames, D. J. Org. Chem. 2007, 72, 1476-1479; (c) Roger, J.; Doucet, H. Adv. Synth. Catal. 2009, 351, 1977-1990; (d) Roger, J.; Doucet, H. Tetrahedron 2009, 65, 9772-9781; (e) Roger, J.; Pozgan, Doucet, H. Adv. Synth. Catal. 2010, 352, 696-710; (f) Fu, H. Y.; Chen, L.; Doucet, H. J. Org. Chem. 2012, 77, 4473-4478.

11. For recent examples of 2-arylbenzothiophenes synthesis: Duan, Z.; Ranjit, S.; Liu, X. Org. Lett. 2010, 12, 2430-2433.

12. (a) Mosrin, M.; Monzon, G.; Bresser, T.; Knochel, P. Chem. Commun. 2009, 5615-5617; (b) Zhao, H.; Dankwardt, J. W.; Koenig, S. G.; Singh, S. P. Tetrahedron Lett. 2012, 53, 166169.

13. (a) Chelucci, G.; Baldino, S.; Ruiu, A. J. Org. Chem. 2012, 77, 9921-9925; (b) Uozumi, Y.; Nakai, Y. Org. Lett. 2002, 4, 2997-3000; (c) Biro, A. B.; Kotschy, A. Eur. J. Org. Chem. 2007, 1364-1368. 